\title{
Wave-front Transformation with Gradient Metasurfaces
}

\author{
Nasim Mohammadi Estakhri and Andrea Alù \\ Department of Electrical and Computer Engineering, \\ The University of Texas at Austin, Austin, Texas 78712, USA \\ (Received 9 July 2015; revised manuscript received 16 August 2016; published 14 October 2016)

\begin{abstract}
Relying on abrupt phase discontinuities, metasurfaces characterized by a transversely inhomogeneous surface impedance profile have been recently explored as an ultrathin platform to generate arbitrary wave fronts over subwavelength thicknesses. Here, we outline fundamental limitations of passive gradient metasurfaces in molding the impinging wave and show that local phase compensation is essentially insufficient to realize arbitrary wave manipulation, but full-wave designs should be considered. These findings represent a critical step towards realistic and highly efficient conformal wave manipulation beyond the scope of ray optics, enabling unprecedented nanoscale light molding.
\end{abstract}

DOI: 10.1103/PhysRevX.6.041008

Subject Areas: Metamaterials, Nanophysics, Optics

\section{INTRODUCTION}

Devising physical systems that grant full control of the distribution of electromagnetic (EM) waves has been an emerging area of research in the past decades. Photonic crystals [1] and metamaterials [2] represent the two major milestones in this direction, and several unusual wave phenomena have been put forward based on these artificially engineered structures, most notably negative refraction and perfect lensing, improved optical fibers, extreme wave localization, and invisibility cloaks [3-6]. One of the long-standing challenges in this context, originally considered at radio frequencies (rf), is to come up with practical and efficient techniques to arbitrarily mold the emerging wave front of an antenna or a localized source. Conventional methods to solve this problem date back to glass lenses and prisms, which rely on engineering the gradual accumulation of phase delay as the wave propagates in the device, reshaping the scattered wave front and beam profile at will. In the context of metamaterials, transformation optics has become a paradigm to realize arbitrary wave manipulation in volumetric devices [7-9]. On top of the unavoidable loss induced along the propagation path, often substantial when metamaterial components are considered [10], these devices suffer from bulky profiles and are typically at least several wavelengths thick. A relevant question we address in this paper is whether it would be possible to realize a transformation platform similarly capable of molding wave propagation at will, but

\footnotetext{
*To whom correspondence should be addressed. alu@mail.utexas.edu

Published by the American Physical Society under the terms of the Creative Commons Attribution 3.0 License. Further distribution of this work must maintain attribution to the author(s) and the published article's title, journal citation, and DOI.
}

with a much thinner and low-profile geometry, in other words, over a metasurface.

At rf, low-profile devices for wave-front patterning are available in the form of arrays of printed antennas, also known as reflect and transmit arrays, which are used to modify the spatial distribution of reflected and transmitted waves over deeply subwavelength thicknesses [11-13]. In contrast to the gradual transformation of the wave in a volumetric component, the variations in the spacing and size of the printed antennas create a transversely inhomogeneous surface impedance profile that imposes an effective field discontinuity, controlling the transverse phase distribution of the wave as it propagates through the surface. Such impedance surfaces can be designed with extremely subwavelength thickness, forming the foundation for a transformation-optics paradigm over two dimensions. Passive printed antenna arrays are at the basis of planar microwave lenses and mirrors that can replace inconvenient bulky mirrors in reflector antennas and, at the same time, can enable fully electric beam steering by exploiting appropriately engineered surface resonances. The simplicity of surface-based wave manipulation at $\mathrm{rf}$ has inspired scientists to extend these concepts to shorter wavelengths, up to the infrared and visible spectrum, using artificial arrays of subwavelength polarizable particles, or metasurfaces [14-17]. In analogy to rf patterned surfaces, optical metasurfaces can be fruitfully modeled with a local averaged surface impedance [18]. However, different from their $\mathrm{rf}$ counterparts, metasurfaces can largely benefit from plasmonic effects, allowing their surface constituents, i.e., nanoantennas [19], to resonate over spatial scales much smaller than the free-space wavelength, providing a route to much larger control of the transverse spatial resolution. Plasmonic metasurfaces have enabled the concept of reflect and transmit arrays to shorter wavelengths, under the assumption that a suitably tailored transverse phase discontinuity profile imparted over an ultrathin surface may 
(a)

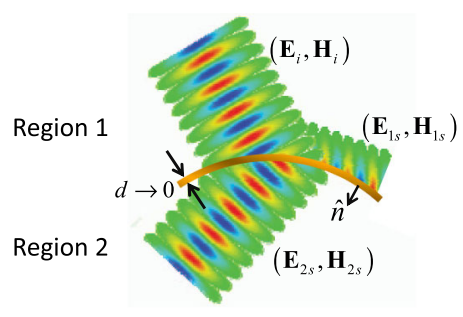

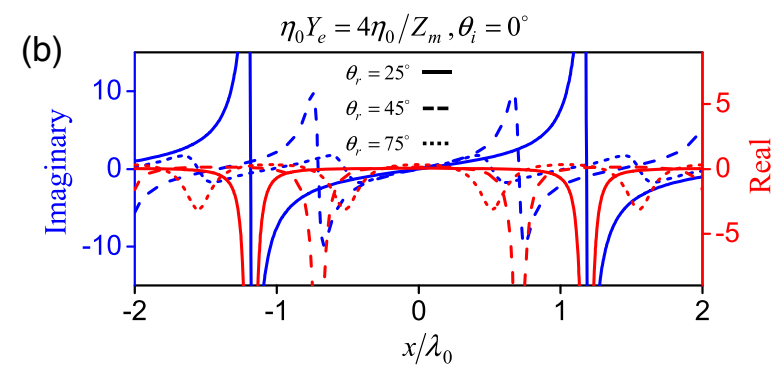
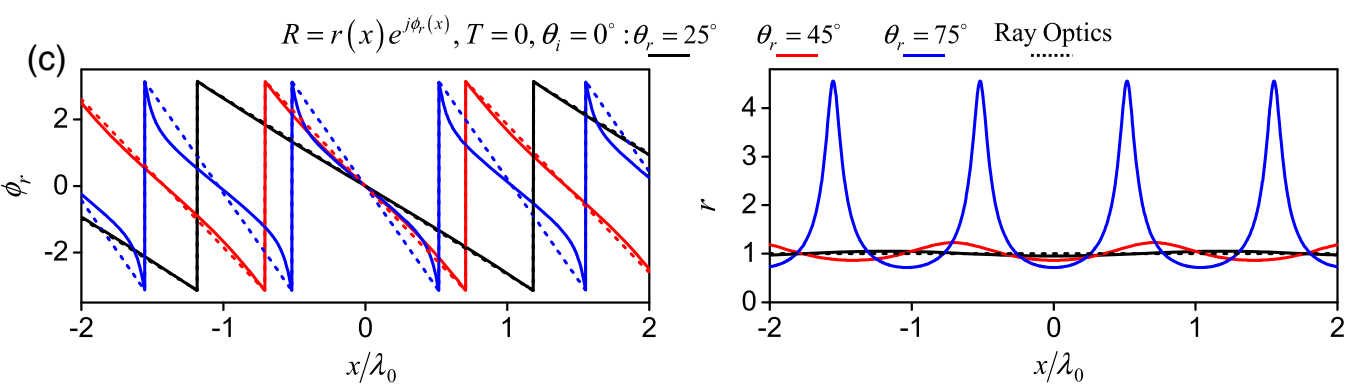

FIG. 1. (a) An arbitrary incident wave front is transformed into the desired scattering profile employing a transversely inhomogeneous metasurface with local distribution of loss and gain. (b) Metasurface impedance profile and (c) local reflection coefficient $R(x)=$ $r(x) e^{j \phi_{r}(x)}$ required to convert a normally incident wave $\left(\theta_{i}=0 \mathrm{deg}\right)$ into refracted waves at $\theta_{r}=25,45,75 \mathrm{deg}$. In each example, the amplitude of the reflected plane wave is $\left|A_{r}\right|=\sqrt{\cos \theta_{i}} / \sqrt{\cos \theta_{r}}$ to ensure that total incident and reflected powers are equal towards the normal direction. The real component of the surface admittance in (b) and nonunitary local reflection amplitudes in (c) indicate the requirement of loss or gain modulation of the surface.

redirect an impinging wave toward a new direction, depending on the lateral phase gradient [20-22]. The abrupt phase shift introduced by nanoantennas is exploited to compensate for the phase difference between the incident and desired wave profile, e.g., linear, circular, and hyperbolic scattering phases, to create tilted waves, vortex beams, and focusing lenses. The prospect of full control on the distribution of the scattered wave with a single ultrathin patterned surface has created excitement in the scientific community, and the initial proposals have now been extended to different frequency ranges, and numerous optimized surface elements have been studied to provide efficient (i.e., with almost unitary amplitude) full phase coverage in both reflection and transmission scenarios [23-27].

In this article, we raise the fundamental question of to what extent a phase gradient on a metasurface is sufficient to guarantee ultimate control of its scattering signature. First, it is obvious that, different from volumetric metamaterials, a metasurface can transform a wave front to another one only provided that both fields are solutions of the source-free wave equation in the background medium. With rigorous treatment, in this paper we show that it is not possible to funnel the incident wave front into an arbitrary solution of choice if we are limited to using passive metasurfaces, and even basic functionalities, such as wave deflection, have fundamental limitations on the overall efficiency of the transformation process in the ultrathin limit. Second, we show that the optimal phase distribution that maximizes the transformation efficiency in the case of a passive surface drastically deviates from the simple phase correction recipe stemming from ray optics, which has been widely used in the recent literature. For instance, ray optics predicts a linear phase distribution along the surface to realize a beam steering metasurface. However, we show that the complete wave optics picture requires the ideal abrupt phase discontinuity to be nonlinear. The deviation, arising from local impedance mismatch on the surface, is particularly important for extreme cases, such as large-angle beam deflection or near-field focusing, for which metasurfaces can outperform conventional volumetric devices or gratings.

\section{BEAM STEERING WITH METASURFACES}

We start by considering the general problem of EM wave interactions with a transversely inhomogeneous metasurface of arbitrary profile, as shown in Fig. 1(a). We assume that the surface thickness is deeply subwavelength, $d / \lambda_{0} \rightarrow 0$, where $\lambda_{0}=2 \pi / k_{0}$ is the free-space wavelength and $k_{0}$ is the corresponding wave number. The subwavelength thickness of the structure allows us to describe it, in a local sense, by equivalent transverse surface electric and magnetic currents $\mathbf{J}_{s}$ and $\mathbf{M}_{s}$, forming local electric admittance $\mathbf{J}_{s}=\underline{\underline{Y}}_{e}(\mathbf{r}) \cdot \mathbf{E}_{t}$ and magnetic impedance $\mathbf{M}_{s}=\underline{\underline{\mathbf{Z}}}_{m}(\mathbf{r}) \cdot \mathbf{H}_{t}$ tensors, related to the tangential components of the local fields over the surface [14,18]. For simplicity, in the following we assume that the symmetries of the problem allow us to consider scalar impedances $Y_{e}$ and $Z_{m}$ for the excitation of interest. It is possible to characterize the surface also by its local reflection $\left[R=r(\mathbf{r}) e^{j \phi_{r}(\mathbf{r})}\right] \quad$ and $\quad$ transmission $\quad\left[T=t(\mathbf{r}) e^{j \phi_{t}(\mathbf{r})}\right]$ 
coefficients, defined assuming a uniform surface built with such local impedances and excited at normal incidence. As shown in Eq. (1), these quantities are directly related to $Y_{e}$ and $Z_{m}$ [28], and $\phi_{r}$ and $\phi_{t}$ are the phase distributions imparted on the reflected and transmitted waves at the metasurface interface, respectively. A lossless ultrathin surface has locally $r^{2}+t^{2}=1$ or, equivalently, $Y_{e}$ and $Z_{m}$ are purely imaginary,

$$
\begin{aligned}
R & =-\frac{2\left(\eta_{0}^{2} Y_{e}-Z_{m}\right)}{\left(2+\eta_{0} Y_{e}\right)\left(2 \eta_{0}+Z_{m}\right)}, \\
T & =-\frac{\left(-2+\eta_{0} Y_{e}\right)}{\left(2+\eta_{0} Y_{e}\right)}+\frac{2\left(\eta_{0}^{2} Y_{e}-Z_{m}\right)}{\left(2+\eta_{0} Y_{e}\right)\left(2 \eta_{0}+Z_{m}\right)} .
\end{aligned}
$$

In order to highlight the potential and limitations of a gradient metasurface for wave transformation, we first consider an ideal planar metasurface whose elements can be engineered to locally provide unitary transmission, zero reflection, and full control on the transmission phase, so that $\phi_{t}(\mathbf{r})$ can take any value between 0 and $2 \pi$ over the surface. This implies that the metasurface can, in principle, impart any phase profile to the transmitted wave, with $100 \%$ local efficiency. In order to transform a normally incident plane wave, $\mathbf{E}_{i}=\hat{y} E_{0} e^{j k_{0} z}$, into an obliquely transmitted wave, $\mathbf{E}_{t}=\hat{y} E_{0} e^{j k_{0}\left[-\sin \left(\theta_{t}\right) x+\cos \left(\theta_{t}\right) z\right]}$, propagating toward the angle $\theta_{t}$ in the $x z$ plane (anomalous refraction, a common target for gradient metasurfaces), the available literature has so far considered designs based on phase compensation, which requires that the metasurface provides a constant phase gradient $\phi_{t}(x)=$ $-k_{0} \sin \left(\theta_{t}\right) x$ [20-22]. This is the basis of the so-called "generalized Snell's law of refraction," which allows challenging the usual refraction response at a transversely homogeneous interface.

We show in the following that this picture is inherently approximate, as it does not consider the relevance of impedance matching in the scattering process [29]. Changing the refraction angle from normal to oblique implies a different ratio of the transverse components of electric and magnetic fields on the surface, which in turn requires that the local transmission coefficient $t$ should be different for local tangential electric and magnetic fields. In other words, contrary to the common assumptions in recent metasurface works, a passive-lossless surface whose sole role is to imprint a locally engineered linear momentum to the scattered wave necessarily fails to generate a plane wave tilted toward an arbitrary direction with unitary efficiency, and the impedance mismatch is expected to grow for steeper angles. This is consistent with earlier papers analyzing linear gradient metasurfaces (see, e.g., Ref. [30]), which have commonly found a degradation of coupling efficiency as the steering angle grows away from the normal.

It is possible to rigorously derive the impedance requirements for a single ultrathin metasurface to transform an arbitrary impinging wave front $\left(\mathbf{E}_{i}, \mathbf{H}_{i}\right)$ into the scattered waves $\left(\mathbf{E}_{1 s}, \mathbf{H}_{1 s}\right)$ in region 1 and $\left(\mathbf{E}_{2 s}, \mathbf{H}_{2 s}\right)$ in region 2, with the only assumption that all three field distributions are valid solutions of source-free Maxwell's equations in the respective regions. The averaged induced current distributions on the metasurface should be suitably designed to compensate for the field discontinuity across the interface [28], and surface admittance and impedance need to satisfy the boundary condition at each point on the surface [23,31],

$$
\begin{aligned}
\hat{n} \times\left.\left(\mathbf{H}_{2}-\mathbf{H}_{1}\right)\right|_{S} & =\left.\frac{1}{2} Y_{e}\left(\mathbf{E}_{2 t}+\mathbf{E}_{1 t}\right)\right|_{S}, \\
\hat{n} \times\left.\left(\mathbf{E}_{2}-\mathbf{E}_{1}\right)\right|_{S} & =-\left.\frac{1}{2} Z_{m}\left(\mathbf{H}_{2 t}+\mathbf{H}_{1 t}\right)\right|_{S} .
\end{aligned}
$$

Assuming that impinging and scattered fields are known, Eq. (2) formulates the exact isotropic metasurface boundary condition that allows converting the impinging wave front into the desired reflected and transmitted waves, and the formulation may straightforwardly be extended to the anisotropic case when polarization coupling is present $[32,33]$. The subscript $t$ represents the tangential field components in each region. The extracted electric admittance and magnetic impedance from Eq. (2) can be directly used in Eq. (1) to calculate the local reflection and transmission coefficients along the metasurface.

In the particular case of a wave-bending metasurface, the incident and scattered fields are plane waves propagating in specified directions. As a basic example, we look into the case of redirecting an impinging plane wave toward the desired direction in reflection, with zero transmission. Incident and scattered fields are linearly polarized transverse-electric (TE) plane waves with wave vectors $\mathbf{k}_{i}=k_{0}\left[-\sin \left(\theta_{i}\right) \hat{x}+\cos \left(\theta_{i}\right) \hat{z}\right]$ and $\mathbf{k}_{r}=-k_{0}\left[\sin \left(\theta_{r}\right) \hat{x}+\right.$ $\left.\cos \left(\theta_{r}\right) \hat{z}\right]$ in region 1 , whereas the total fields are enforced to be zero in region 2 [Fig. 1(a)]. The metasurface is located in the $x z$ plane, as shown in the inset of Fig. 3(b). Following Eq. (2), the required electric surface admittance and surface magnetic impedance to realize this scattering signature are

$$
\begin{aligned}
Y_{e} & =\left.2 \frac{\hat{x} \cdot\left(\mathbf{H}_{i}+\mathbf{H}_{1 s}\right)}{\hat{y} \cdot\left(\mathbf{E}_{i}+\mathbf{E}_{1 s}\right)}\right|_{z=0} \\
& =\frac{2}{\eta_{0}} \frac{\cos \left(\theta_{i}\right) e^{-j k_{0} \sin \left(\theta_{i}\right) x}-A_{r} \cos \left(\theta_{r}\right) e^{-j k_{0} \sin \left(\theta_{r}\right) x}}{e^{-j k_{0} \sin \left(\theta_{i}\right) x}+A_{r} e^{-j k_{0} \sin \left(\theta_{r}\right) x}}, \\
Z_{m} & =\left.2 \frac{\hat{y} \cdot\left(\mathbf{E}_{i}+\mathbf{E}_{1 s}\right)}{\hat{x} \cdot\left(\mathbf{H}_{i}+\mathbf{H}_{1 s}\right)}\right|_{z=0} \\
& =2 \eta_{0} \frac{e^{-j k_{0} \sin \left(\theta_{i}\right) x}+A_{r} e^{-j k_{0} \sin \left(\theta_{r}\right) x}}{\cos \left(\theta_{i}\right) e^{-j k_{0} \sin \left(\theta_{i}\right) x}-A_{r} \cos \left(\theta_{r}\right) e^{-j k_{0} \sin \left(\theta_{r}\right) x}},
\end{aligned}
$$

in which $A_{r}$ is the amplitude of the electric field in the reflected plane wave, normalized to the incident one.

Based on the general condition in Eq. (2), it is possible to show that total reflection is possible if and only if $Y_{e} Z_{m}=4$ (see Appendix A), which is indeed satisfied by 
Eq. (3). Figure 1(b) shows the required distribution of $Y_{e}$ and $Z_{m}$ along the metasurface that ensures anomalous reflection with unitary power efficiency, plotted for normal incidence and various reflection angles. Following the periodicity of the incident and scattered waves, the attained surface holds a superlattice periodicity $X$ along the $x$ axis, as shown in Fig. 1(b), related to the incident and reflection angles by $X=\left|\lambda_{0} /\left(\sin \theta_{r}-\sin \theta_{i}\right)\right|$ [24]. The reflected wave, hence, corresponds to the first diffraction order of the gradient metasurface. To ensure unitary efficiency, the relative amplitude of the reflected wave should also be $\left|A_{r}\right|=\sqrt{\cos \theta_{i}} / \sqrt{\cos \theta_{r}}$ in Eq. (3) (see Appendix B), so that incoming and outgoing power flows are equal; i.e., $\hat{z} \cdot \mathbf{P}_{\text {incident }}=-\hat{z} \cdot \mathbf{P}_{\text {reflected }}$.

Interestingly, the required surface to achieve unitary efficiency always involves local loss and gain [passive and active portions correspond to simultaneous positive and negative values of both $\operatorname{Re}\left[Y_{e}\right]$ and $\operatorname{Re}\left[Z_{m}\right]$ in Fig. 1(b)], and it is not passive in a local sense, consistent with Huygens transmit arrays introduced in Ref. [23]. This is expected, since the total power emerging right above the metasurface, $\operatorname{Re}\left[\hat{z} \cdot \mathbf{P}_{\text {total }}(x)\right]=-1 /\left.2 \operatorname{Re}\left[E_{y} H_{x}^{*}\right]\right|_{z \rightarrow 0^{+}}$, can be explicitly calculated from the required field distribution, superposition of the impinging and reflected waves,

$$
\begin{aligned}
\operatorname{Re}\left[\hat{z} \cdot \mathbf{P}_{\text {total }}(x)\right]= & \frac{E_{0}^{2}}{2 \eta_{0}}\left(\left|A_{r}\right|^{2} \cos \left(\theta_{r}\right)-\cos \left(\theta_{i}\right)\right. \\
& +\left|A_{r}\right|\left[\cos \left(\theta_{r}\right)-\cos \left(\theta_{i}\right)\right] \\
& \left.\times \cos \left\{\angle A_{r}+k_{0}\left[\sin \left(\theta_{i}\right)-\sin \left(\theta_{r}\right)\right] x\right\}\right),
\end{aligned}
$$

whose value oscillates from positive to negative values along $x$. The only exception is $\theta_{r}= \pm \theta_{i}$, or accordingly for the specular and retroreflection, for which $\operatorname{Re}\left[\hat{z} \cdot \mathbf{P}_{\text {total }}(x)\right]=0$ everywhere. This is expected, as the incident and reflected local impedances are matched for these special cases, and, interestingly, a passive-lossless metasurface is sufficient to fully transform the incident wave [see Eq. (B5)]. Apart from this condition, the optimal [34] surface described by Eq. (2) necessarily requires that the local power absorption or gain oscillates around zero along $x$. Interestingly, the requirements on distributed loss or gain modulation cannot be relaxed in reflecting metasurfaces that preserve the polarization state of the wave, even through introduction of surface bianisotropy. Physically, this limitation also stems from the local impedance mismatch on the metasurface, which remains intact for polarization preserved reflection. In general, i.e., for refracting metasurfaces and/or polarization conversion, bianisotropy may relax the local amplitude modulation along the surface $[35,36]$. It follows that the only way to keep a unitary conversion efficiency towards the desired direction with a steering ultrathin metasurface is to locally absorb and pump a portion of the incident power in different regions within the superlattice (equivalent to nonlocal operation). At the same time, the surface remains globally lossless, in the sense that the averaged net power supplied by the surface, $\bar{P}_{\text {surface }}=-(1 / 2 S) \int_{S} Y_{e}^{*}\left|\mathbf{E}_{t}\right|^{2} d s+\int_{S} Z_{m}\left|\mathbf{H}_{t}\right|^{2} d s$, is identically zero, as required by the choice of $\left|A_{r}\right|$ to ensure unitary power efficiency. This raises interesting connections with the field of parity-time symmetry and balanced loss and gain [37-39], a feature that has been recently shown to open exciting opportunities in optics. Highly efficient steering metasurfaces appears to also require a specific balance of loss and gain. Details on power absorption calculations and conditions on the passivity of the surface are found in Appendix B.

After transforming the derived impedance profile into local reflection and transmission coefficients using Eq. (1), we find the local phase and amplitude profiles required to create the desired wave-front deflection with an ultrathin metasurface:

$$
\begin{aligned}
R(x) & =r(x) e^{j \phi_{r}(x)} \\
& =-1+\left.\frac{2 \hat{y} \cdot\left(\mathbf{E}_{i}+\mathbf{E}_{1 s}\right)}{\hat{y} \cdot\left(\mathbf{E}_{i}+\mathbf{E}_{1 s}\right)+\eta_{0} \hat{x} \cdot\left(\mathbf{H}_{i}+\mathbf{H}_{1 s}\right)}\right|_{z=0}, \quad T=0 .
\end{aligned}
$$

These are visualized in Fig. 1(c), which shows the local reflection phase $\phi_{r}(x)$ and amplitude $r(x)$ at the metasurface, highlighting alternating regions with local loss and gain, with $r>1$ and $r<1$, respectively. While unitary power conversion efficiency is possible only using local gain and loss elements, it is interesting that the phase requirements are also quite different from the simple linear distribution predicted by ray optics.

Equations (3)-(5) and Fig. 1 show the first important conclusion of our analysis: efficient beam steering towards arbitrary angles with an ultrathin surface cannot be achieved using passive-lossless linear phase profiles. Interestingly, when the anomalous reflection angle is close to specular reflection, linear phase compensation along the surface provides a very good approximation for the optimal surface. However, as we increase the deflection angle, and we get into the regime in which metasurfaces can outperform conventional gratings in terms of efficiency, thanks to their subwavelength control of the transverse resolution, our rigorous solution significantly deviates both in amplitude and phase from the linear phase approximation commonly used in the literature.

In the following, we focus on passive metasurfaces in order to avoid the requirement of active elements, which may be difficult to realize and may introduce challenging stability limitations. First, it is interesting to notice that, while the previous analysis shows that balanced gain and loss is necessary to achieve ideal energy steering with a metasurface, it may still be possible to route all the scattered energy towards a preferred direction with proper 
(a)

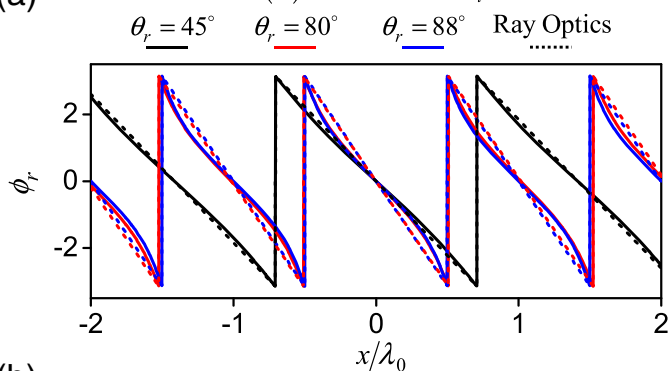

(b)

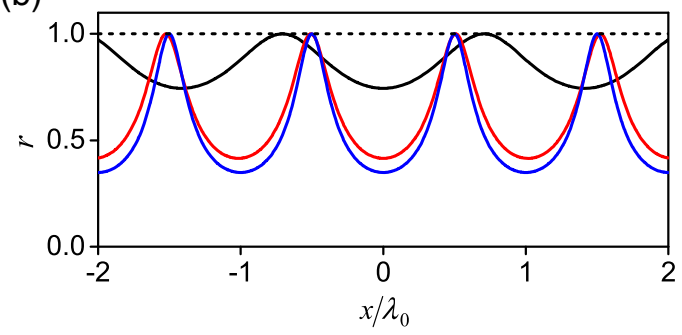

(c)

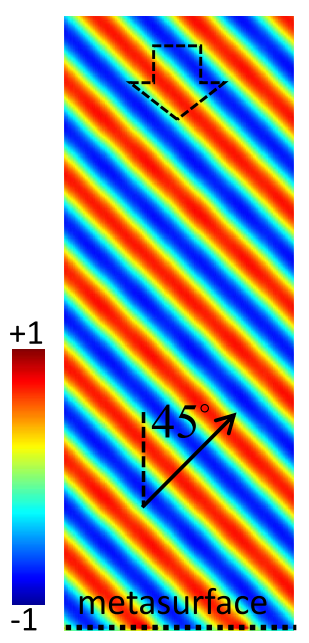

(d)

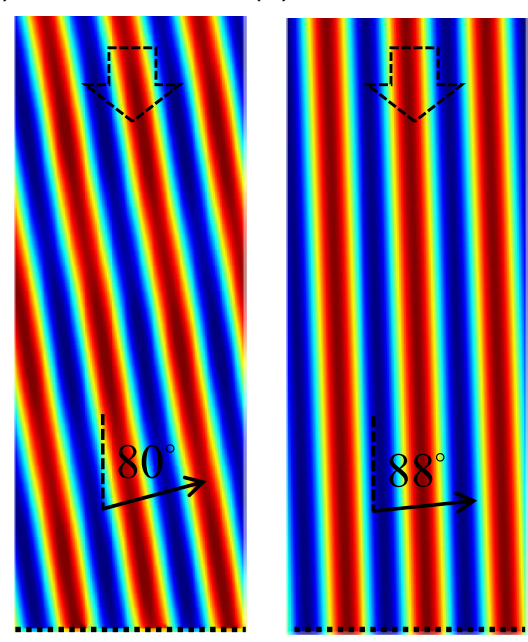

FIG. 2. (b) Local reflection coefficient $R(x)=r(x) e^{j \phi_{r}(x)}$ required to convert a normally incident wave $\left(\theta_{i}=0\right.$ deg) into (a) refracted waves at $\theta_{r}=45,80,88 \mathrm{deg}$ while preserving its amplitude, i.e., $\left|A_{r}\right|=1$. Distribution of the scattered magnetic field $H_{z}$, for $\theta_{i}=0$ and (c) $\theta_{r}=45, d=\lambda_{0} / 20$, (d) $\theta_{r}=80, d=\lambda_{0} / 20$, (e) $\theta_{r}=88, d=\lambda_{0} / 200$, for the passive-lossy surfaces illustrated in (a) and (b). Panels (c)-(e) correspond to overall efficiencies of $70.6 \%, 17.3 \%$, and $3.5 \%$, respectively. All plots are normalized to the amplitude of the incident magnetic field and the metasurfaces are realized in an all-electric grounded setup, as shown in the inset of Fig. 3(b).

design. The requirement of unitary power efficiency implies that the power density steered towards the desired angle grows as $\cos \theta_{i} / \cos \theta_{r}$, simply following the projection of the wave vector to the surface normal. If we allow the scattered power in the normal direction to be less than the incident one, the surface will provide a net absorption, $\operatorname{Re}\left(\bar{P}_{\text {surface }}\right)<0$, up to the point for which the ideal surface would exhibit only lossy components (i.e., at all points $r \leq 1$ ), implementing a distributed loss pattern over the surface similar to Ref. [40]. Interestingly, we rigorously show in Appendix B that selective coupling to the desired diffraction order can be achieved with a passive-lossy surface, provided that the relative amplitude of the reflected wave is equal to or lower than $\left|A_{r}\right|_{\max }=\min \left[1, \cos \left(\theta_{i}\right) /\right.$ $\cos \left(\theta_{r}\right)$ ], valid for both TE and TM waves. This indicates that, using Eq. (3), it is possible to design a passive-lossy surface that steers a normally incident beam exclusively to an arbitrary direction of choice, while preserving the amplitude of electric and magnetic fields, i.e., $\left|A_{r}\right|=1$. This surface would necessarily lose a portion of the impinging power since the outgoing power is less than the impinging one, but scattering to other diffraction orders can be made identically zero at the cost of efficiency. Figures 2(a) and 2(b) illustrate the required reflection phase and amplitude for three reflection angles in this scenario. As expected, the local reflection amplitude varies along the surface; yet, its maximum value is limited to unity, $r_{\max }=1$. In accordance with our previous discussion, as the reflection angle increases, the required local reflection phase along the surface departs from the linear approximation.
There is a wide range of metasurface configurations that may physically implement high-resolution phase elements in various setups, from microwave to infrared and optical frequencies. Plasmonic and dielectric nanoantennas, composite particles, printed circuits, multilayered meta-atoms, and wire antennas [20,23-26,41-43] provide fertile ground for local phase (and also amplitude) manipulation. The desired reflection profile $R(x)$ and zero transmission may also be implemented without relying on a series impedance distribution $Z_{m}(x)$, which would inherently require magnetic effects, by simply using a gradient nonmagnetic surface admittance backed by a ground plane [24,30,31], as schematically shown in the inset of Fig. 3(b) (see Appendix C for more discussion on all-electric implementation of Huygens surfaces). This would significantly facilitate the synthesis of the desired response in a practical design, especially in the optical range for which magnetic responses are typically weak. Based on such implementation for the calculated local reflections in Figs. 2(a) and 2(b), Figs. 2(c)-2(e) show the corresponding normal component of the magnetic field distribution (which is present only in the reflected beam), showing full coupling towards the desired direction, and zero scattering toward unwanted directions. The synthesis of amplitude modulation along the surface, as in Fig. 2(b), may be achieved either by varying locally absorbing elements or by using anisotropic inclusions and modulating the crosspolarization coupling or loss, as recently suggested in Refs. [40,44], to simultaneously realize desired amplitude and phase modulation with a metasurface. 
(a)

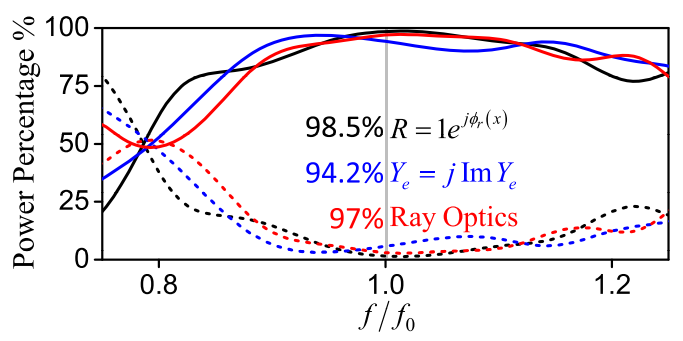

(c)

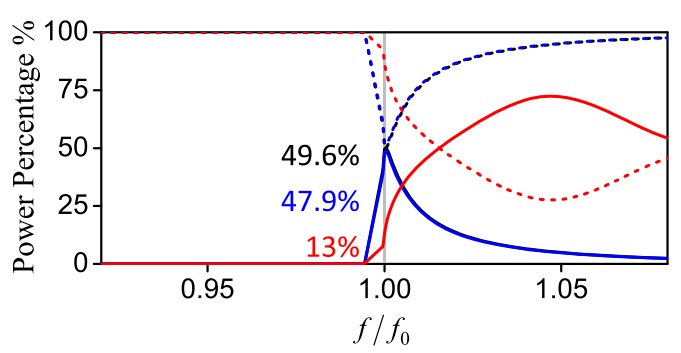

(b)

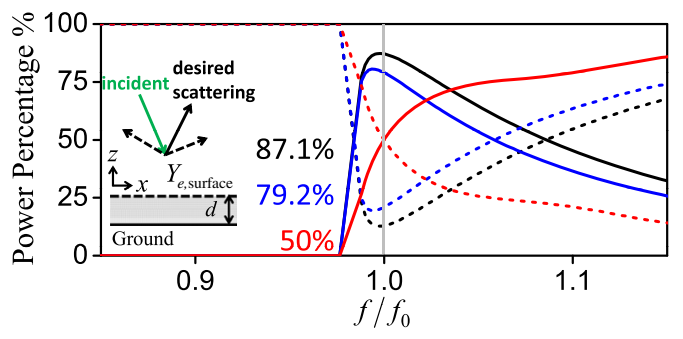

(d)

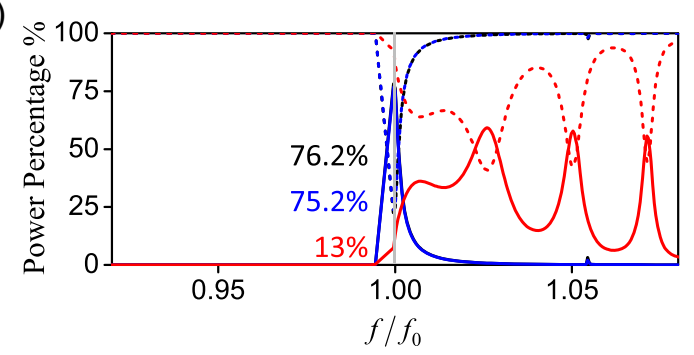

FIG. 3. Frequency variation of the power reflected into the desired diffraction order for $\theta_{i}=0$ and (a) $\theta_{r}=45, d=\lambda_{0} / 20$, (b) $\theta_{r}=80, d=\lambda_{0} / 20$, (c) $\theta_{r}=88, d=\lambda_{0} / 50$, (d) $\theta_{r}=88, d=\lambda_{0} / 200$. The inset of (b) shows the geometry of an all-electric grounded metasurface. Solid lines indicate the percentage of power coupled into the desired direction. All examples correspond to passive-lossless metasurfaces with different approximations indicated in the inset of (a).

The designs of Fig. 2 provide the maximum coupling efficiency to achieve exclusive scattering in a desired direction of choice with a passive-lossy metasurface. As described in the caption, the efficiency may become drastically low for large steering angles (an efficiency of $3.49 \%$ is available for a steering angle of $88 \mathrm{deg}$ ), which may not be desirable or practical. These results, however, show again the relevance of going beyond the ray-optics approximation, and properly tailor amplitude and phase of the local reflection coefficient to design efficient gradient metasurfaces. Yet, in several applications it may be important to maximize the amount of power coupled towards the desired direction with a lossless surface, even though this may require coupling a small portion of it towards other diffraction orders [45]. Since we proved that no passive metasurface can achieve unitary power efficiency beam steering in near and far field, next we explore what impedance profile is needed to boost its overall coupling. In such a case, loss over the surface should be avoided, and we focus therefore on lossless impedance profiles.

Considering normal incidence, Fig. 3 shows the simulated power distribution scattered from lossless surfaces designed to steer towards $\theta_{r}=45,80,88 \mathrm{deg}$ at the design frequency $f_{0}$, while the response considers the natural frequency dispersion of the grounded metasurface related to the finite distance between the surface and ground plane. Solid lines indicate the percentage of incident power coupled into the desired diffraction order, while dashed lines indicate the portion of power scattered into other orders, based on the superlattice periodicity of the beam steering surface [Figs. 1(b) and 1(c)]. The black lines refer to the case in which the structure is designed to impart the phase profile extracted from Eq. (5), as in Fig. 1(c), while the local reflection amplitude is unitary. As we discuss above, the imparted phase gradient in this case is different from the phase difference between incident and reflected waves, especially for steep deflection angles. The blue curves refer to the case in which the metasurface is designed by simply discarding the real part of the impedance profiles in Fig. 1(b) (and thus the reflection amplitude is once more unitary). Finally, the red curves refer to the case when the linear phase predicted from ray optics is imprinted over the surface, as in most conventional metasurface designs. Details on the metasurface design and scattering calculation are provided in Appendix B.

Quite predictable from our previous discussions, for a deflection angle $\theta_{r}=45 \mathrm{deg}$, the constraints on loss and gain are moderate, and all cases provide very large conversion efficiencies. Yet, as the deflection angle increases, the linear phase approach fails to follow the desired scattering profile, and at $\theta_{r}=80$ and $88 \mathrm{deg}$, only $50 \%$ and $13 \%$ of power is coupled to the desired directions, respectively. With the phase profile retrieved from Eq. (5), on the other hand, around $87 \%$ and $50 \%$ efficiency can be attained. Pushing down the thickness of the structure, the reflecting surface better mimics a metasurface, and the coupling efficiency to $\theta_{r}=88 \mathrm{deg}$ grows to over $76 \%$, as shown in Fig. 3(d). Despite the clear difference between the approximate phase profile and our approach, both profiles are quite smoothly varying [Fig. 1(c)] and the coupling efficiency is predicted to be robust to spatial discretization of the surface profile, even for large deflection angles, as we study in the next section. Clearly, for larger 

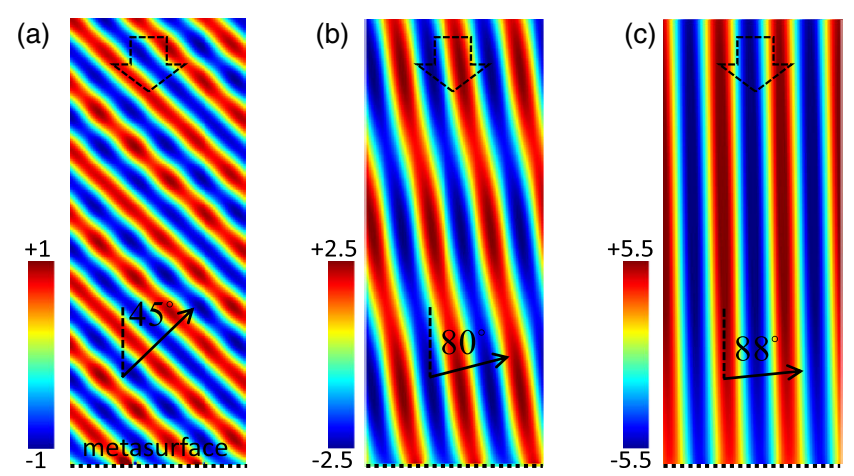

FIG. 4. Distribution of the scattered magnetic field $H_{z}$, for $\theta_{i}=0$ and (a) $\theta_{r}=45, d=\lambda_{0} / 20$, (b) $\theta_{r}=80, d=\lambda_{0} / 20$, (c) $\theta_{r}=88, d=\lambda_{0} / 200$, for the lossless approximation scenario. All plots are normalized to the amplitude of the incident magnetic field, and panels (a)-(c) correspond to overall power conversion efficiencies of $98.5 \%, 87.1 \%$, and $76.2 \%$, respectively. The loss and gain profile of the optimal metasurface is approximated by its local reflection phase, i.e., $R(r)=1 e^{j \phi_{r}(x)}$.

angles the superlattice footprint shrinks and proper quantization requires smaller surface granularities to maintain high efficiency. While no passive ultrathin surface may provide unitary power efficiency, a design that considers the impedance mismatch of the deflected wave makes it indeed possible to steer a significant portion of the impinging wave towards an arbitrary angle, in both near and far fields, well beyond the limits of conventional gratings.

The striking features of the proposed wave-shaping metasurfaces may be better appreciated by investigating the field distributions plotted in Fig. 4, corresponding to the designs in Figs. 3(a) and 3(b), and 3(d). Still relying on lossless gradient metasurfaces, and without ad hoc optimization but simply following Eq. (2) and neglecting the amplitude modulation, we are able to efficiently rotate the incident wave vector (normal) toward extremely oblique angles, with minimal unwanted scattering. The key factor to achieve these close-to-optimal efficiencies is to go beyond the ray approximation and linear phase gradients, and instead to engineer the phase distribution following the previous formulation.

Imparting the phase profile extracted from Eq. (5) also allows us to go beyond the maximum efficiencies attainable from multimode Huygens metasurfaces $[29,42]$. Multimode metasurfaces rely on the presence of one or more additional scattering modes to ensure surface passivity, and they may suffer from low efficiencies and a large number of evanescent modes close to the metasurface, particularly at large deflection angles. Designing the metasurface in the near field, however, allows us to create efficient wave-shaping metasurfaces that maintain their performance even in the proximity of the surface. This property allows us to achieve complex near-field operations, such as near-field focusing, as discussed in Sec. V.

\section{PHYSICAL IMPLEMENTATION AND EFFECTS OF DISCRETIZATION OF THE IDEAL METASURFACE PROFILE}

The nonlinear, relatively fast-varying phase profiles in Fig. 1(c) raise important questions regarding the stability of the response to surface discretization, which may be necessary in a practical implementation. To investigate this effect, we design, based on the previous formulation, a metasurface reflect array to convert a normally incident plane wave into a plane wave propagating towards $\theta_{r}=75 \mathrm{deg}$, with minimal coupling to spurious modes in near and far field. The reflection phase and amplitude of this surface are shown as solid blue lines in Figs. 1(c) and 1(d). Each period of the surface is then divided into $N_{Q}$ segments, where we set $N_{Q}=4,8,16$, and we enforce unitary local reflection coefficient, implementing a passive lossless approximation of the ideal metasurface. Figure 5(a) illustrates the spatial distribution of the surface electric admittance $Y_{\text {esurface }}$ over one period of the grounded metasurface [shown in Fig. 1(b)], calculated for $N_{Q}=8$ and $d=\lambda_{0} / 20$. The corresponding continuous and quantized local reflection phases on the surface are also shown in Fig. 5(b). Analogous to the examples provided in Sec. II,

(a)

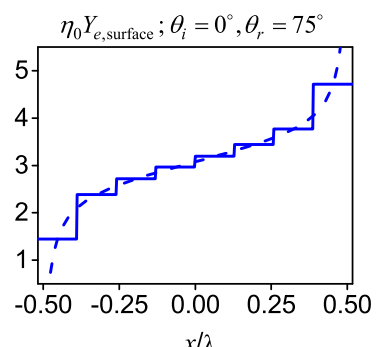

(b) $R=e^{j \phi_{r}(x)}, T=0, \theta_{i}=0^{\circ}, \theta_{r}=75^{\circ}$

(c)

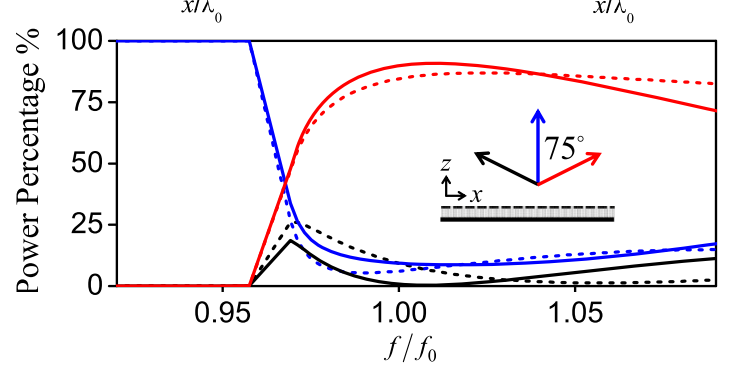

FIG. 5. (a) Spatial distribution of admittance profile and (b) local reflection coefficient of the grounded metasurface designed to redirect a normal TE incident plane wave towards $\theta_{r}=75 \mathrm{deg}$. The admittance layer is at $d=\lambda_{0} / 20$ distance from the ground plane and the passive, lossless approximation is considered, i.e., $R(x)=1 e^{j \phi_{r}(x)}$. The amplitude of the reflected plane wave is set at $\left|A_{r}\right|=1 / \sqrt{\cos 75^{\circ}}$ in Eq. (3). Solid lines show the discretized profiles for $N_{Q}=8$ and dashed lines correspond to the original continuous pattern. (c) Frequency variation of the power reflected into different diffraction orders of the quantized metasurface. Red, blue and black lines correspond to $\theta_{r}=75,0,-75 \mathrm{deg}$ at the center design frequency $f=f_{0}$. Dashed lines indicate the coupling efficiency of the continuous metasurface. 
full-wave simulations of the periodic setup are used to evaluate the percentage of power coupled toward each Floquet harmonic, shown in Fig. 5(c). For easier comparison, we also report the efficiency of the continuous (nonquantized) surface in the same panel with dashed lines.

We observe over 94\%, 90\% [shown in Fig. 5(c)], and $87 \%$ coupling efficiency toward the desired direction, respectively, for $N_{Q}=4,8$, and 16. Quite interestingly, the original continuous gradient surface provides $85 \%$ overall efficiency and the quantized profiles appear to be closer to the best possible profile for a passive lossless wave-bending metasurface. This improved performance is associated with the elimination of singular impedance values due to the discretization of the impedance profile, and it appears quite favorable for experimental implementation of these surfaces. In addition, the stability of the response to a rough discretization implies that, with the implementation of the local impedances in a realistic setup, one can expect increasingly improved performance for metasurfaces designed based on our analytical solution in comparison to those designed based on the ray-optics approximation.

We demonstrate these findings by implementing the structure studied in Fig. 5 in a realistic setup, designed for operation at $1 \mathrm{GHz}$. Each of the eight admittance or phase steps in Figs. 5(a) and 5(b) are realized using four individual inductor-capacitor (LC) series resonators, placed on a 10 -mm-thick $\left(=\lambda_{0} / 30\right)$ Eccostock $® P P$ foam [46], as shown in Fig. 6(a). Each period of the gradient metasurface, thus, contains 32 surface resonators in the $x$ direction and one resonator in the $y$ direction. The LC components are electrically connected through metallic patches placed on the foam and the entire structure is grounded at the back surface. To design the gradient metasurface, we first calculate the local reflection phase on the top surface of each building block for commercially available chip inductors and capacitors [47], in a periodic setup. Subsequently, the element values are appropriately selected in accordance with Fig. 5(b). Columns two and three in Table I list the final design parameters. For comparison, we also repeat the same procedure to design another metasurface based on the ray-optics approximation, i.e., using a linear local reflection phase (columns four and five in Table I), as commonly done in conventional gradient metasurface designs.

The overall performance for the two cases is evaluated through full-wave simulations, and the percentage of total incident power coupled into each propagating Floquet harmonic is shown in Fig. 6(b). As expected, the performance of the metasurface designed based on our analytical approach significantly outperforms the metasurface designed based on the linear phase approximation [shown with dashed lines in Fig. 6(b)], using similar discretization. Specifically, at the center frequency, our technique provides around $89 \%$ coupling from a normally incident wave to the $n=1$ Floquet mode, while ray optics provides only around $59 \%$ efficiency. It is quite fascinating that, although we implement each admittance or phase step with only four elements, our realistic metasurface design provides comparable performance to a quantized surface implemented

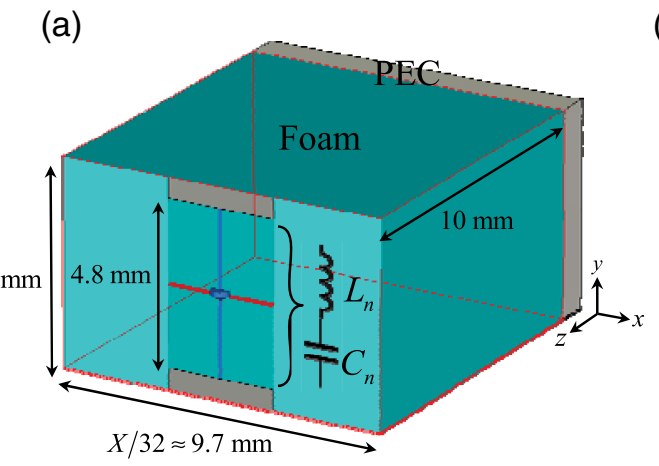

(b)

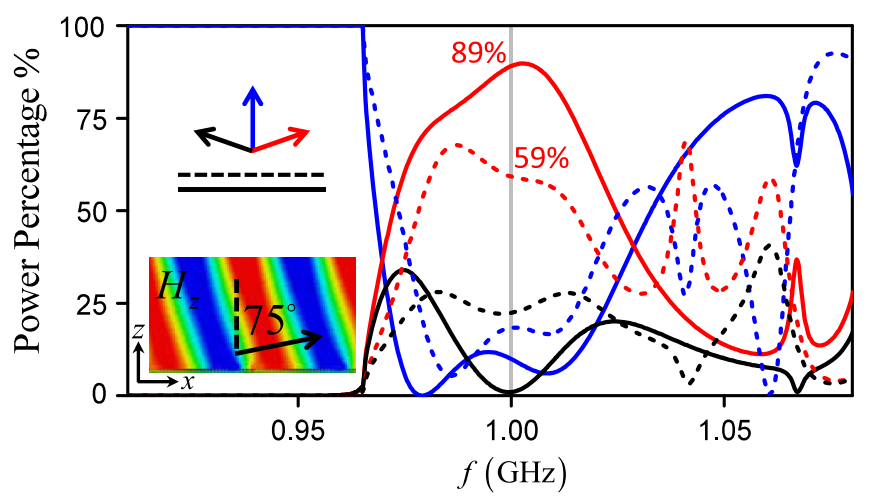

FIG. 6. (a) Schematic of the microwave building block to implement gradient metasurfaces designed to redirect a normal incident plane wave toward $\theta_{r}=75 \mathrm{deg}$ at $1 \mathrm{GHz}$. Each block consists of a 10-mm-thick Eccostock®PP foam with relative permittivity $\varepsilon_{r}=1.06$ placed on a perfect electric conducting (PEC) layer. Elements are assumed to be passive and lossless, and the local reflection phase on top of each element is controlled by varying the surface capacitors and inductors. Thirty-two blocks are utilized in order to implement one supercell period of the intended gradient metasurfaces, and $X=\left|\lambda_{0} /\left(\sin \theta_{r}-\sin \theta_{i}\right)\right| \approx 310.6$ mm. (b) Distribution of the reflected power toward different Floquet harmonics. Solid lines indicate the performance of the gradient metasurface designed based on the passive, lossless approximation of our analytical solution, i.e., $R(x)=1 e^{j \phi_{r}(x)}$, and the dashed lines demonstrate analogous results for the metasurface designed based on the ray-optics approximation, i.e., $R(x)=1 e^{-j 2 \pi x / X}$. Except for the LC surface components listed in Table I, all physical properties of the two metasurfaces are similar. Red lines indicate the percentage of power successfully redirected in the direction of the first Floquet harmonic, i.e., $\theta_{r}=75 \mathrm{deg}$ at $f=1 \mathrm{GHz}$. The inset shows a time snapshot of $H_{z}$ at $1 \mathrm{GHz}$ for the metasurface designed based on our approach, demonstrating the clean scattered wave profile even in close proximity to the surface. The field amplitude is normalized to the incident plane wave. 
TABLE I. Design parameters for the wave-bending metasurface to redirect a normal TE incident plane wave toward $\theta_{r}=75$ deg. Metasurface consists of 32 surface series resonators and is designed at $1 \mathrm{GHz}$. The lossless approximation is considered here as $R(x)=1 e^{j \phi_{r}(x)}$, and the ray optics approximation is implemented with the linear local phase $R(x)=1 e^{-j 2 \pi x / X}$. All local phases are calculated on top of the surface. Capacitor and inductor values are specified in $\mathrm{pF}$ and $\mathrm{nH}$, respectively.

\begin{tabular}{lcccc}
\hline \hline $\begin{array}{l}\text { Element types } \\
\text { and numbers }\end{array}$ & $\begin{array}{c}\text { Local phase } \\
\text { for } R(x)=1 e^{j \phi_{r}(x)}\end{array}$ & $\begin{array}{c}\text { Element values } \\
\text { for } R(x)=1 e^{j \phi_{r}(x)}\end{array}$ & $\begin{array}{c}\text { Local phase for } \\
R(x)=1 e^{-j 2 \pi x / X}\end{array}$ & $\begin{array}{c}\text { Element values } \\
\text { for } R(x)=1 e^{-j 2 \pi x / X}\end{array}$ \\
\hline$\left(C_{1-4}, L_{1-4}\right)$ & $117.1^{\circ}$ & $(1.1,10)$ & $157.5^{\circ}$ & $(0.1,2.2)$ \\
$\left(C_{5-8}, L_{5-8}\right)$ & $69.5^{\circ}$ & $(1.7,4.7)$ & $112.5^{\circ}$ & $(1.5,4.3)$ \\
$\left(C_{9-12}, L_{9-12}\right)$ & $39.7^{\circ}$ & $(1.3,10)$ & $67.5^{\circ}$ & $(1.8,3.9)$ \\
$\left(C_{13-16}, L_{13-16}\right)$ & $13^{\circ}$ & $(1.9,4.3)$ & $22.5^{\circ}$ & $(1.2,12)$ \\
$\left(C_{17-20}, L_{17-20}\right)$ & $-13^{\circ}$ & $(1.9,4.7)$ & $-22.5^{\circ}$ & $(1.3,11)$ \\
$\left(C_{21-24}, L_{21-24}\right)$ & $-39.7^{\circ}$ & $(1.6,7.5)$ & $-67.5^{\circ}$ & $(1.8,6.2)$ \\
$\left(C_{25-28}, L_{25-28}\right)$ & $-69.5^{\circ}$ & $(1.5,9.1)$ & $-112.5^{\circ}$ & $(1.5,10)$ \\
$\left(C_{29-32}, L_{29-32}\right)$ & $-117.1^{\circ}$ & $(2.2,4.7)$ & $-157.5^{\circ}$ & $(3.6,2.2)$ \\
\hline \hline
\end{tabular}

with ideal surface profile (Fig. 5). In this regard, the predicted efficiency of an ideal quantized surface with similar substrate material and overall thickness is $90 \%$ for our approach and $59 \%$ for the ray optics solution.

Following these results, it is expected that, with appropriate implementation of the surface impedance, and even with quite rough discretization of $N_{Q}=4,8$, it is possible to design highly efficient metasurfaces for wave-front transformation. For instance, for the aforementioned example of bending a normally incident beam toward $75 \mathrm{deg}$, at $\lambda_{0}=500 \mathrm{~nm}$ and $N_{Q}=4$, each surface element is approximately $130 \mathrm{~mm}$ wide, which can be practically implemented using subwavelength high-index nanoparticles. Over visible wavelengths, the strong mutual interactions between adjacent particles (which can be calculated analytically for dissimilar surface components [48]), and the enhanced local density of states combined with low absorption, make high-index dielectric metasurfaces an excellent choice, with the intriguing prospect of tunability $[43,49,50]$. Another particularly interesting scenario is the case of graphene ribbons or patches, which may be able to model one-atom-thick tunable impedance sheets at terahertz frequencies. Graphene-based metasurfaces may be an ideal implementation of the structure envisioned in Fig. 3(b), and may be fabricated with deeply subwavelength resolution and rigorously designed based on our accurate analytical model [51]. For instance, at $1 \mathrm{THz}$ and for $N_{Q}=16$, each surface element is over $19 \mu \mathrm{m}$ wide, which is well above the state-of-the-art fabrication resolution for graphene metasurfaces [52,53].

\section{METASURFACE COUPLERS}

An extreme example of beam steering is the case in which we aim at converting a propagating wave into a bound state, as in a surface coupler, which corresponds to the case of complex $\theta_{r}$ in our previous formulation. As $\theta_{r}$ approaches $90 \mathrm{deg}$, the reflection wave vector $\vec{k}_{r}=-k_{0} \hat{x}$ will be solely along the tangential direction, with transverse momentum equal to the free-space wave number. Beyond this point, the wave vector is larger than $k_{0}$, which can be conveniently modeled by a complex reflection angle $\theta_{r}=90+j\left|\alpha_{r}\right|$. This problem has been approached in the literature using gratings or linear phase gradient metasurfaces, providing the required momentum mismatch between incident and guided waves [54-58]. Artificial symmetry breaking in the scattering properties of the graded surface, along with proper optimization of the coupling structure, has been exploited to enhance the coupling efficiency $[59,60]$. However, following the previous discussion, we can rigorously explore the conditions to achieve optimal coupling with a gradient metasurface, and we expect a linear phase gradient to be far from optimal. Similar to Eq. (3), given the incident and scattered wave profiles, the required surface impedances to couple a normally incident plane wave into a guided mode along the $x$ direction with transverse momentum $\beta_{x}=-k_{0} \cosh \left(\alpha_{r}\right)$ equals

$$
Y_{e}=\frac{2}{\eta_{0}} \frac{k_{0}+A_{r} k_{z} e^{j k_{x} x}}{A_{r} k_{0} e^{j k_{x} x}+k_{0}}, \quad Z_{m}=4 / Y_{e},
$$

in which $\beta_{z}=j\left|\sqrt{\beta_{x}^{2}-k_{0}^{2}}\right|$. The required surface impedance is in this case complex, with alternating regions of loss and gain depending on the relative amplitude of the guided wave $A_{r}$. Notice that this infinite surface coupler is an extreme example, inherently ill posed, presented here to confirm the generality of our proposed theory: while the gradient metasurface supports the desired guided mode along the surface, the normal incident power $\hat{z} \cdot \mathbf{P}_{\text {incident }}$ cannot contribute to the power propagating in the lateral direction, since the power flowing along the surface is constant for a guided mode over an infinite periodic structure. Indeed, in the infinite metasurface coupler described by Eq. (6), the net power absorbed by the surface $\operatorname{Re}\left(-\bar{P}_{\text {surface }}\right)$ over each superlattice period is equal to the incident power, independent from $A_{r}$ (see Appendix D), consistent with power conservation. The surface inhomogeneous loss or gain profile is then responsible for generating the desired guided mode under the excitation of such a 
(a)

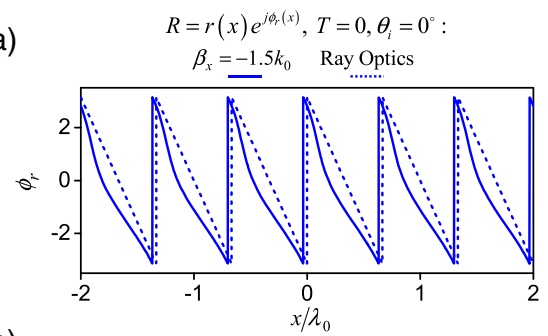

(b)

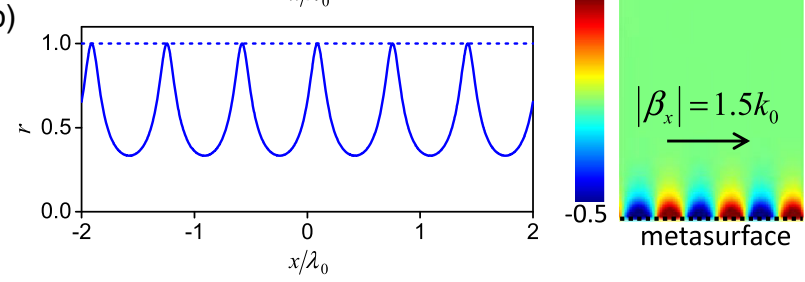

FIG. 7. (a), (b) Metasurface local reflection coefficient $R(x)=r(x) e^{j \phi_{r}(x)}$, required to convert a normally incident wave $\left(\theta_{i}=0 \mathrm{deg}\right)$ into a guided wave with $\beta_{x}=-1.5 k_{0}$, in a passivelossy metasurface, i.e., $\left|A_{r}\right|=k_{0} /\left|\beta_{x}\right|$. (c) Distribution of the scattered magnetic field $H_{z}$, for $\theta_{i}=0$ and $\beta_{x}=-1.5 k_{0}$, $d=\lambda_{0} / 20$, for the all-passive surface illustrated in (a) and (b). The field is normalized to the amplitude of the incident magnetic field.

plane wave. For a beam of finite cross section, on the other hand, we can expect efficient coupling to the desired modal profile, using Eq. (2).

Interestingly, even limiting ourselves to passive surfaces, the required surface scattering phase is far from linear, as shown in Fig. 7(a) for $\beta_{x}=-1.5 k_{0}$. Here, the relative amplitude of the guided mode is chosen to assure passivity of the surface, following the previously discussed approach [Fig. 7(b)]. It can be shown that the gradient coupling surface is locally passive and lossy over all lateral points, as long as $\left|A_{r}\right|<k_{0} /\left|\beta_{x}\right|$, as shown in Appendix D. A linear scattering phase approximation may allow coupling a portion of incident power to a guided wave with parallel wave vector [56-58], similar to a conventional grating. However, it fails to provide the optimal surface profile to maximize the coupling efficiency [55]. Conversely, as shown in Fig. 7(c), the rigorous formulation described here is capable of creating a pure secondary guided wave with the desired distribution using a patterned metasurface, and zero coupling into other scattering orders.

We conclude this section noting that this approach can be extended to the practical problem of the design of finite-sized surface couplers. In this case, the excitation field is not an infinite plane wave, but the finite incident beam profile that excites the coupler. At the same time, the desired scattered wave should be a gradually growing surface wave that adiabatically matches the mode profile of the fed waveguide. Quite distinct from infinite couplers, the total power carried by the incident wave is finite in this case, and it matches the total guided mode power. In addition, the normal component of the incident wave directly feeds the guided mode. Interestingly, a ray-optics approach is unable to provide any adaptation on the size or profile of the coupler based on
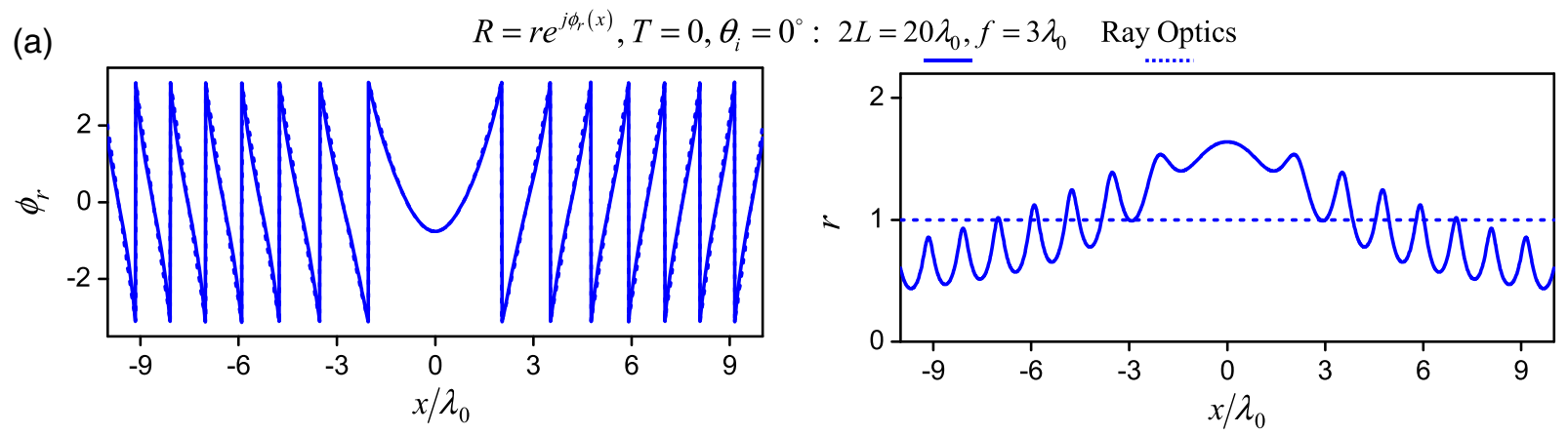

(b)

$R=r e^{j \phi_{r}(x)}, T=0, \theta_{i}=0^{\circ}: 2 L=8 \lambda_{0}, f=0.25 \lambda_{0} \quad$ Ray Optics
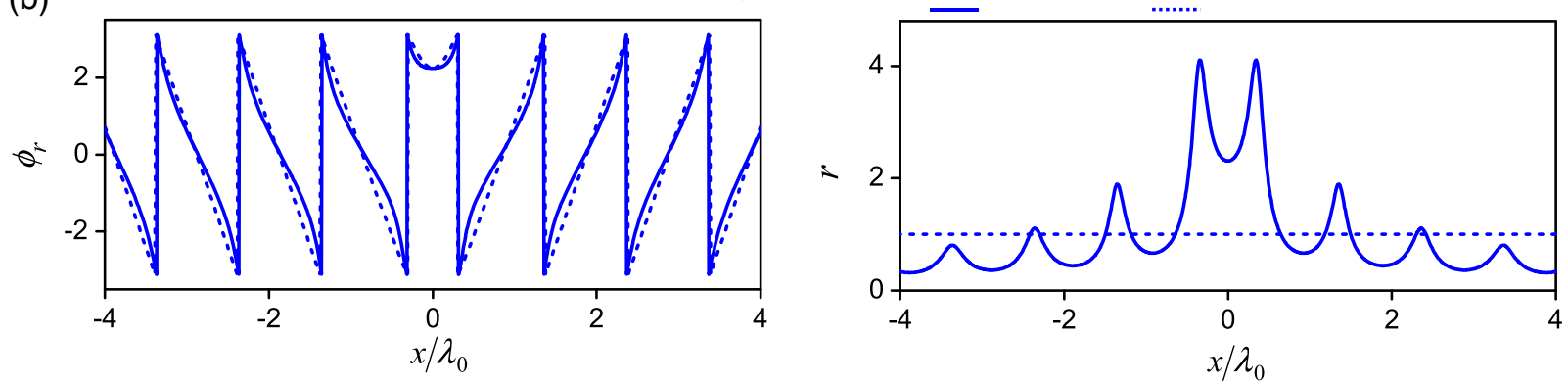

FIG. 8. Comparison between the scattering properties of ideal (solid lines) and ray-optics-based (dashed lines) metasurface reflecting lenses with local distribution of loss and gain and (a) NA $=0.9578$ and (b) NA $=0.9981$. Amplitude and phase of the local reflection coefficient $R(x)=r(x) e^{j \phi_{r}(x)}$ are plotted along the lens surface for (a) $\alpha=\pi$ and (b) $\alpha=\pi / 2$ (see Appendix E). Metasurface lenses are extended between $x=(-L, L)$, excited by a plane wave propagating along the $-z$ direction. 
these specifications, and is thus limited in overall coupling efficiency and in impedance matching to the fed waveguide. Consequently, ray-optics-based couplers are typically limited to low conversion efficiencies, analogous to traditional grating-based couplers [55]. While a specific design of a surface wave coupler goes beyond the scope of this paper, the required surface profile is expected to have amplitude modulation and to enable significantly enhanced coupling efficiencies.

\section{METASURFACE LENSING AND FOCUSING}

The approximations of the ray-optics approach highlighted in the previous section are not limited to wave bending and coupling. Here, we examine the design of an ultrathin planar lens with extreme focusing properties. Reducing the volumetric size of a dielectric lens into a single patterned surface is of great interest in nano-optics
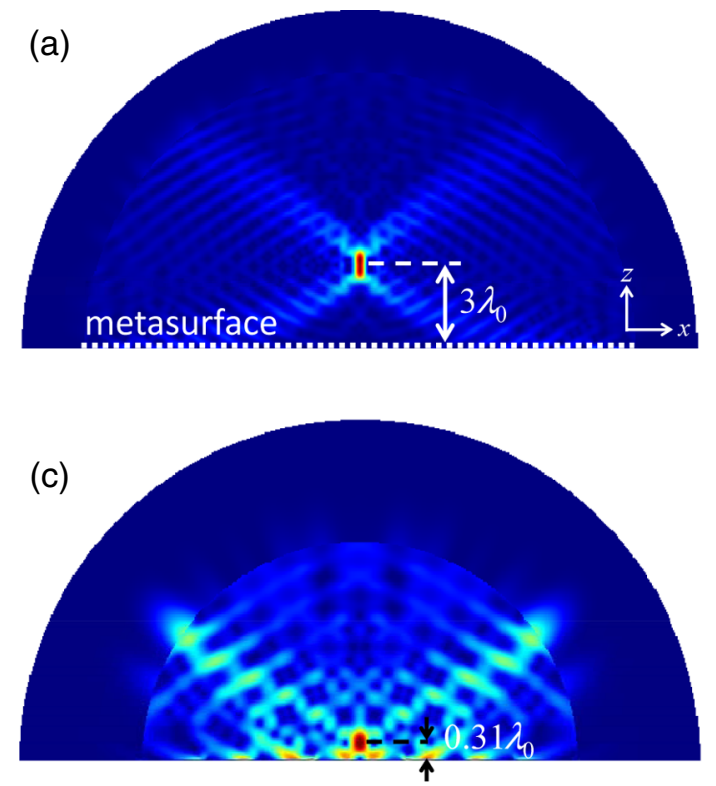

(e)
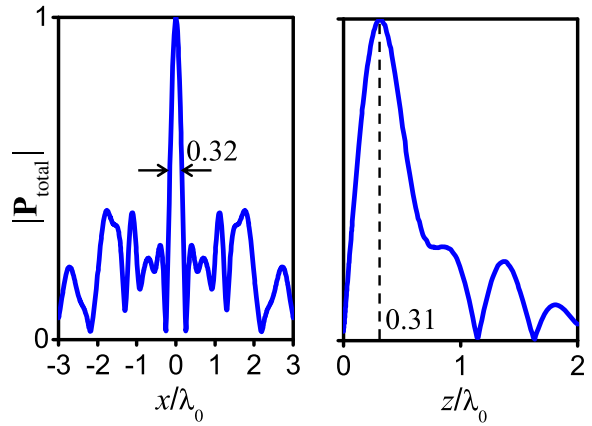

and integrated photonics, and the local phase compensation approach has been incorporated in the designs of numerous flat focusing structures [27,61]. To generate a spherical outgoing phase front from an incident plane wave, ray optics suggests a hyperboloidal phase profile to be imprinted over the surface (i.e., all normally incident rays will be redirected towards the desired direction and collimate at the focal point). However, the previous results suggest that this approach would provide a reasonable performance only in the limit of small numerical apertures (NA), and when the focal point is located far from the lens. Under such a condition, the rays traced from the corner of the lens, which experience the largest local deflection angle, are far from the grazing angle and the phase difference between scattered and incident waves is close to the phase (and amplitude) profile of the optimal surface. For NA $<0.96$, the cone angle of the lens is smaller than 73 deg and, as illustrated in Fig. 8(a), the hyperboloidal
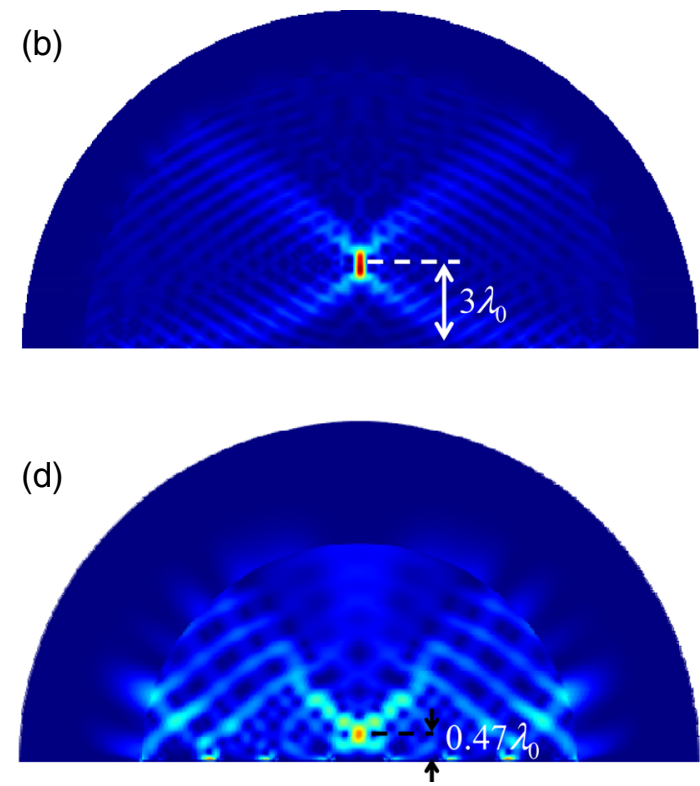

(f)
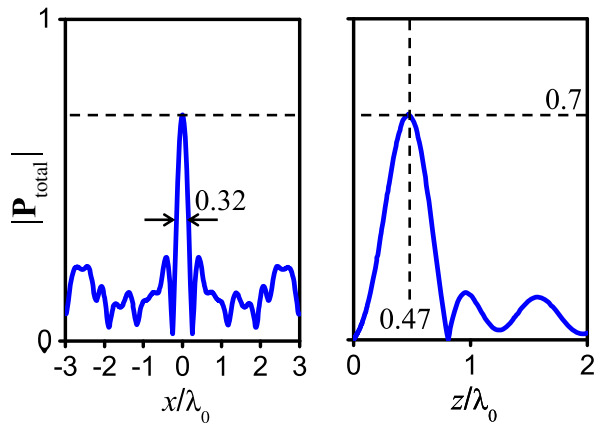

FIG. 9. Comparison between the power distribution in passive-lossless planar lenses with optimal (a), (c) and hyperbolic (b), (d) lateral phase profiles. Plots in the same row have the same color bar, and all metasurfaces have equal thickness, $d=\lambda_{0} / 50$. Imparted local reflection coefficients $R(x)=1 e^{j \phi_{r}(x)}$ are calculated for (a) $\alpha=\pi$ and (c) $\alpha=4.4$ rad (see Appendix E). Metasurface lenses in (a), (b) and (c), (d) are designed to collimate the normally incident plane wave at $f_{1}=3 \lambda_{0}$ and $f_{2}=0.25 \lambda_{0}$, respectively. (e), (f) Field profile along the $x$ and $z$ directions across the focal points corresponding to (c) and (d), respectively. For better comparison, the plots are all normalized to the same value. 
interfacial phase pattern (dashed line) mimics the exact surface profile (solid lines) with about 35-deg error range, while the ideal reflection amplitude oscillates around unity. As we increase the numerical aperture to ranges that are not achievable with conventional diffraction elements, the line shape of the optimal phase drastically deviates from a simple hyperbolic pattern and we find over 70-deg error range for $\mathrm{NA}=0.998$. To design the optimal surface in accordance with Eq. (2), a normally incident plane wave is transformed into an outgoing cylindrical wave. In Appendix E, we determine the optimal amplitude and phase of the secondary wave in each case to ensure unitary focal efficiency; i.e., $\operatorname{Re}\left(\hat{z} \cdot \overline{\mathbf{P}}_{\text {reflected }}\right)=\operatorname{Re}\left(-\hat{z} \cdot \overline{\mathbf{P}}_{\text {incident }}\right)$. As we move toward higher numerical apertures, as expected, the optimal surface requires extremely localized loss and gain segments, and the scattering phase significantly diverges from the hyperboloidal approximation. We stress that these limitations are independent of the resolution of the gradient metasurface, and a surface designed based on local phase compensation inevitably exhibits unwanted spherical aberrations and fails to create focusing effects in the near-field region.

In accordance with the previous results on wave bending, the optimal active or passive surface can be approximated by its passive-lossless counterpart to preserve the ideal phase pattern, and provide an improved passive-lossless lowaberration, high-numerical-aperture lens design, by sacrificing a small portion of the focused energy. This is illustrated in Fig. 9, where we compare two lenses with overall lengths of $20 \lambda_{0}$ and $8 \lambda_{0}$, designed to focus the incident wave at $f_{1}=3 \lambda_{0}$ and $f_{2}=0.25 \lambda_{0}$, with $\mathrm{NA}=0.958$ and $\mathrm{NA}=0.998$, respectively. Both lenses are lossless, and implemented with grounded all-electric metasurfaces, shown in Fig. 3(b). The first column illustrates the power distribution above the reflecting lenses that are designed based on the optimal phase distribution on the surface. The second column shows the power distribution when a hyperbolic phase distribution is imprinted over the surface. As expected, for a small numerical aperture and reasonably large focal distance, ray optics provides a very good approximation of the exact solution, yet it fails to create near-field focusing effects [Fig. 9(d)]. On the contrary, the passive-lossless metasurface lens, designed based on our analytical solution, provides strong near-field focusing effects, as shown in Fig. 9(c). Compared to the ideal lens, the focal point is slightly shifted from the intended position (less than $0.06 \lambda_{0}$ ), toward the $+z$ direction. This minor deviation is associated with the lossless approximation of the ideal lens, as well as the absence of an active drain at the focal point. Quite interestingly, we also find that the half-power beam width of the focal image is similar in both approaches, and it is approximately $0.32 \lambda_{0}$ [Figs. 9(e) and 9(f)].

\section{CONCLUSIONS}

In this paper, we investigate the theoretical limitations and potentials of passive gradient metasurfaces for arbitrary wave manipulation. Our study is based on a rigorous treatment of the wave equation, which allows deriving relevant results for the field of gradient metasurfaces and wave manipulation over a surface. First, we prove that wave transformations over an ultrathin surface, even in their simplest form, e.g., for beam steering, inherently require the presence of balanced loss and gain to achieve unitary efficiency. Then, we derive a bound on the maximum coupling efficiency that allows exclusively coupling to the desired diffraction order, and we derive a path towards maximizing the coupling efficiency to the wave front of choice, showing the inaccuracy of conventional ray-optics approximations commonly used in the literature to realize wave transformations not achievable with conventional diffraction gratings.

We inspect practical examples of anomalous wave deflection, coupling from propagating waves to surface bounded modes, and lenses, showing that passive-lossless metasurfaces following the derived gradient profiles can significantly outperform designs based on conventional design rules derived from ray optics. We further study the effect of surface quantization on the overall performance of the device and provide a realistic implementation of a gradient metasurface at microwave frequencies, designed following our analytical derivation. Our findings confirm a considerable improvement of $30 \%$ to the overall efficiency for an extreme-angle wave-bending metasurface, compared to a similar metasurface designed using the conventional ray-optics approach. For simplicity, here we consider twodimensional reflection scenarios, yet similar restrictions may be derived for three dimensions, polarization coupling surfaces, and transmitting metasurfaces. Our results shed light on the physical limitations of passive metasurfaces and provide a practical route toward highly efficient waveshaping metasurfaces, beyond the extent attainable from current techniques based on ray optics.

\section{ACKNOWLEDGMENTS}

This work was supported by the Welch Foundation with Grant No. F-1802, the Air Force Office of Scientific Research with Grant No. FA9550-13-1-0204, the National Science Foundation and the Simons Foundation.

\section{APPENDIX A: THEORETICAL FORMULATION OF REFLECTING WAVE-SHAPING METASURFACES}

In the first part of the theoretical discussions in Sec. II, we derive suitable magnetoelectric surface impedance profiles to achieve arbitrary wave-front transformation [Eq. (2)]. With the intention of arbitrarily manipulating the reflected wave with zero transmission, Eq. (2) reduces to

$$
\begin{gathered}
\hat{n} \times\left.\left(\mathbf{H}_{1}\right)\right|_{S}=-\left.\frac{1}{2} \underline{\underline{Y}}_{e} \cdot\left(\mathbf{E}_{1 t}\right)\right|_{S}, \\
\hat{n} \times\left.\left(\mathbf{E}_{1}\right)\right|_{S}=\left.\frac{1}{2} \underline{\underline{\mathbf{Z}}}_{m} \cdot\left(\mathbf{H}_{1 t}\right)\right|_{S},
\end{gathered}
$$


with the total fields set at zero inside region 2 . We set the local right-handed coordinate system on the surface as $\left(\hat{n}, \hat{t}_{1}, \hat{t}_{2}\right)$, with $\hat{n}$ indicating the normal unit vector as shown in Fig. 1(a), and $\left(\hat{t}_{1}, \hat{t}_{2}\right)$ are the two orthogonal transverse unit vectors along the surface. In the most general format, the surface may be anisotropic with admittance dyadic profile $\underline{\underline{Y}}_{e}=Y_{e, t_{1}} \hat{t}_{1} \hat{t}_{1}+Y_{e, t_{2}} \hat{t}_{2} \hat{t}_{2}$ and impedance $\underline{\underline{\mathbf{Z}}}_{m}=Z_{m, t_{1}} \hat{t}_{1} \hat{t}_{1}+Z_{m, t_{2}} \hat{t}_{2} \hat{t}_{2}$. Rewriting and decomposing Eq. (A1), we get

$$
\begin{aligned}
& Y_{e, t_{2}}=-2 \frac{\hat{t}_{1} \cdot \mathbf{H}_{1}}{\hat{t}_{2} \cdot \mathbf{E}_{1}}, \\
& Y_{e, t_{1}}=+2 \frac{\hat{t}_{2} \cdot \mathbf{H}_{1}}{\hat{t}_{1} \cdot \mathbf{E}_{1}},
\end{aligned}
$$

Equation (A2) demonstrates the relation $Y_{e, t_{2}} Z_{m, t_{1}}=$ $Y_{e, t_{1}} Z_{m, t_{2}}=4$ between electric and magnetic properties of a general surface operating in reflection mode. In an isotropic surface, this condition simplifies to $Y_{e} Z_{m}=4$, as given in the main text. Under this condition, the local transmission coefficient $T$, as defined in Eq. (1), is also zero along the surface.

\section{APPENDIX B: POWER, EFFICIENCY, AND SCATTERING ANALYSIS OF METAREFLECT ARRAYS}

A homogenous interface, such as the boundary between two plain dielectrics, supports specular reflection. This means that, when illuminated by a plane wave, any percentage of power reflected by such an interface is funneled into the single wave propagating away from the surface in the mirror direction. This wave is highlighted as the $n=0$ arrow in Fig. 10. When the interface is not

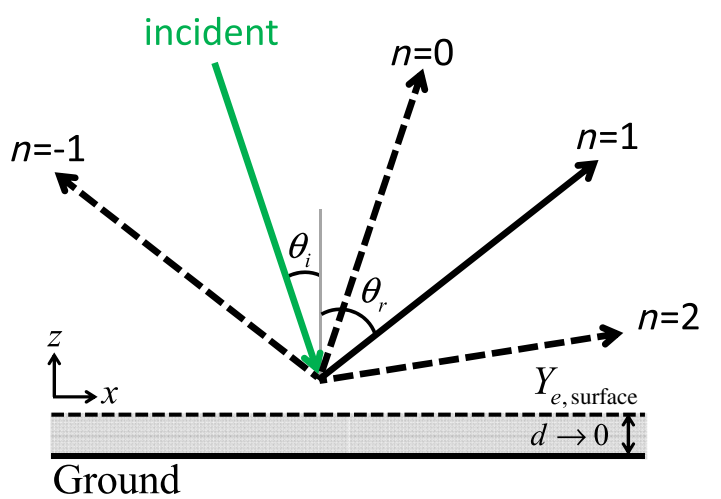

FIG. 10. Trajectory of a plane wave illuminating a periodic gradient metasurface with incident wave vector $\mathbf{k}_{i}=$ $k_{0}\left[-\sin \left(\theta_{i}\right) \hat{x}+\cos \left(\theta_{i}\right) \hat{z}\right]$, and the desired reflected plane wave (solid arrows) with wave vector $\mathbf{k}_{r}=-k_{0}\left[\sin \left(\theta_{r}\right) \hat{x}+\cos \left(\theta_{r}\right) \hat{z}\right]$. Dashed arrows indicate other allowed scattering directions in such configuration. The metasurface is realized as an electric admittance surface mounted in a subwavelength distance from the ground plane. homogenous, the scattered wave is, in general, a combination of all plane waves in the radiation continuum, and for a periodic structure, their integral sum straightforwardly reduces to discrete waves propagating toward specific directions (the discrete diffraction orders). As pointed out in the main text, a surface designed to transform an incident plane wave into another plane wave with different wave vector is inherently periodic. Therefore, one appropriate measure to determine the performance of the surface is to calculate the percentage of power coupled to each of these diffraction orders when illuminating the structure. This is shown in Fig. 3, where we sum over all undesired orders and compare it to the power coupled into the intended one. In our frequency range of interest, each surface couples non-negligible power to a maximum of three orders $n=0, \pm 1$, with $n=+1$ referring to the desired plane wave [solid arrows in Figs. 3(b) and 10].

The required surface properties are extracted from Eqs. (2) and (A1), considering the appropriate distribution of incident and scattered waves as

$$
\begin{aligned}
\left(\mathbf{E}_{i}, \mathbf{H}_{i}\right)= & \left(\hat{y}, \frac{\hat{x} \cos \theta_{i}+\hat{z} \sin \theta_{i}}{\eta_{0}}\right) E_{0} e^{-j \sin \theta_{i} k_{0} x} e^{j \cos \theta_{i} k_{0} z}, \\
\left(\mathbf{E}_{1 s}, \mathbf{H}_{1 s}\right)= & A_{r}\left(\hat{y}, \frac{-\hat{x} \cos \theta_{r}+\hat{z} \sin \theta_{r}}{\eta_{0}}\right) \\
& \times E_{0} e^{-j \sin \theta_{r} k_{0} x} e^{-j \cos \theta_{r} k_{0} z},
\end{aligned}
$$

where the term $A_{r}=\sqrt{\cos \theta_{i}} / \sqrt{\cos \theta_{r}}$ takes care of ensuring that the power reflected in the direction normal to the surface is equal to the impinging power, assuming TE illumination. The averaged total power supplied by the surface can be expressed as $\bar{P}_{\text {surface }}=-(1 /(2 X))$ $\int_{X}\left(Y_{e}^{*}\left|\mathbf{E}_{t}\right|^{2}+Z_{m}\left|\mathbf{H}_{t}\right|^{2}\right) d x \quad$ [62], in which $X=\mid \lambda_{0} /$ $\left(\sin \theta_{r}-\sin \theta_{i}\right) \mid$ is the surface superlattice [24]. After some algebraic simplification, the supplied electric and magnetic power can be written in terms of the incident and scattered angles as

$$
\begin{aligned}
\bar{P}_{\text {surface }, e}= & \bar{P}_{\text {surface }, m} \\
= & -\frac{E_{0}^{2}}{4 \eta_{0} X} \int_{X} \sqrt{\cos \theta_{i} \sec \theta_{r}} \\
& \times\left(\cos \theta_{i} e^{-2 j \pi x / X^{\prime}}-\cos \theta_{r} e^{2 j \pi x / X^{\prime}}\right) d x,
\end{aligned}
$$

both equal to zero $\left[X^{\prime}=\lambda_{0} /\left(\sin \theta_{r}-\sin \theta_{i}\right)\right]$. This property further verifies that the metasurface as a whole does not pump any power into the scattered wave or absorb any part of it, but rather transforms the incident wave to the desired field by proper, locally distributed, balanced absorption and gain. The averaged net power emerging from the surface is equivalently zero in this scenario: 


$$
\begin{aligned}
\bar{P}_{\text {surface }} & =\frac{1}{X} \int_{X}\left[\operatorname{Re}\left(\hat{z} \cdot \mathbf{P}_{\text {total }}\right)_{z \rightarrow 0^{+}}\right] d x \\
& =-\frac{E_{0}^{2}\left[\cos \left(\theta_{i}\right)-\left|A_{r}\right|^{2} \cos \left(\theta_{r}\right)\right]}{2 \eta_{0}}=0 .
\end{aligned}
$$

Equation (B3) also verifies that for $\left|A_{r}\right|=\sqrt{\cos \theta_{i}} / \sqrt{\cos \theta_{r}}$, the net power generated by the surface is zero and the wave-bending efficiency is $100 \%$.

As we discuss in the main text, the reflection amplitude may be chosen so that all surface components are passive (no gain portions), yet we couple just to the desired diffraction order, given that we allow absorbing a portion of the incident energy. The local passivity of the metasurface can be enforced considering either its local admittance, i.e., $\forall x, \operatorname{Re}\left[Y_{e}(x)\right.$ and $\left.Z_{m}(x)\right]>0$, or the local emerging power, i.e., $\forall x, \operatorname{Re}\left(\hat{z} \cdot \mathbf{P}_{\text {total }}\right)_{z \rightarrow 0^{+}}<0$. The first condition implies that, if the surface possesses any resistive component, it must be positive to avoid local power generation. The second condition, equivalently, requires that at no point along the surface the total power flows toward the outgoing direction from the surface. This means that, locally, the metasurface is either lossless [corresponding to $r=1$ points in Fig. 2(b)] or absorptive [corresponding to $r<1$ points in Fig. 2(b)]. Following Eq. (B1), the local emerging power on the surface is found as [Eq. (3) in the main text]

$$
\begin{aligned}
\operatorname{Re}\left(\hat{z} \cdot \mathbf{P}_{\text {total }}\right)_{z \rightarrow 0^{+}}= & \frac{E_{0}^{2}}{2 \eta_{0}}\left(\left|A_{r}\right|^{2} \cos \left(\theta_{r}\right)-\cos \left(\theta_{i}\right)\right. \\
& +\left|A_{r}\right|\left[\cos \left(\theta_{r}\right)-\cos \left(\theta_{i}\right)\right] \\
& \left.\times \cos \left\{\angle A_{r}+k_{0}\left[\sin \left(\theta_{i}\right)-\sin \left(\theta_{r}\right)\right] x\right\}\right) .
\end{aligned}
$$

Enforcing $\operatorname{Re}\left(\hat{z} \cdot \mathbf{P}_{\text {total }}\right)_{z \rightarrow 0^{+}}<0$ and solving Eq. (B4), the maximum acceptable reflection amplitude is found to be $\left|A_{r}\right|_{\max }=\min \left[1, \cos \left(\theta_{i}\right) / \cos \left(\theta_{r}\right)\right]$. The conversion efficiency of the surface under this condition may be found by calculating the incident and reflected power along the $z$ direction:

$\eta=\frac{\operatorname{Re}\left(\hat{z} \cdot \mathbf{P}_{\text {reflected }}\right)}{\operatorname{Re}\left(-\hat{z} \cdot \mathbf{P}_{\text {incident }}\right)}=\frac{\cos \theta_{r}}{\cos \theta_{i}}\left|A_{r}\right|^{2}=\min \left(\frac{\cos \theta_{r}}{\cos \theta_{i}}, \frac{\cos \theta_{i}}{\cos \theta_{r}}\right)$,

consistent with the reciprocity theorem [63]. A similar argument holds for TM waves, where we have

$$
\begin{aligned}
\left(\mathbf{E}_{i}, \mathbf{H}_{i}\right)= & \left(-\hat{x} \cos \theta_{i}-\hat{z} \sin \theta_{i}, \frac{\hat{y}}{\eta_{0}}\right) E_{0} e^{-j \sin \theta_{i} k_{0} x} e^{j \cos \theta_{i} k_{0} z} \\
\left(\mathbf{E}_{1 s}, \mathbf{H}_{1 s}\right)= & A_{r}\left(\hat{x} \cos \theta_{r}-\hat{z} \sin \theta_{r}, \frac{\hat{y}}{\eta_{0}}\right) \\
& \times E_{0} e^{-j \sin \theta_{r} k_{0} x} e^{-j \cos \theta_{r} k_{0} z}
\end{aligned}
$$

with $A_{r}=\sqrt{\cos \theta_{i}} / \sqrt{\cos \theta_{r}}$. The general format of power, as presented in Eqs. (B3)-(B4), also holds for the TM polarization of incident and scattered waves with the same upper bounds on the scattering amplitude.

\section{APPENDIX C: ALL-ELECTRIC IMPLEMENTATION OF HUYGENS METASURFACES}

We implement all of our designed configurations following the method exploited in Refs. [24,31], exclusively based on a grounded electric surface admittance, as shown in Fig. 10. It has been proved that in the limit of subwavelength thickness, $d / \lambda_{0} \rightarrow 0$, a grounded surface with $Y_{e, \text { surface }}=Y_{e} / 2+j \cot \left(k_{0} d\right) / \eta_{0}$ is equivalent to a reflecting magnetoelectric interface [31]. The structures are then simulated in COMSOL Multiphysics 4.4 in a periodic setup to calculate the percentage of power coupled to each Floquet harmonic. No frequency dispersion is embedded in $Y_{e \text {,surface }}$; i.e., $Y_{e \text {,surface }}$ is assumed constant over the entire spectral range. After analytically calculating the required surface admittance, reflection amplitude, and reflection phase, we approximate the ideal metasurface with two different lossless surfaces. First, the real part of the surface admittance is simply ignored; i.e., $Y_{e \text {,surface }}$ is replaced with $j \operatorname{Im}\left[Y_{e, \text { surface }}\right]$. Second, passive $Y_{e, \text { surface }}$ is evaluated so that the surface supports the reflection phase as the ideal surface, but with a unitary amplitude, i.e., $R=1 e^{j \phi_{r}(x)}$ :

$$
Y_{e, \text { surface }}=\frac{1-e^{j \phi_{r}(x)}+j\left(1+e^{j \phi_{r}(x)}\right) \cot k_{0} d}{\eta_{0}\left(1+e^{j \phi_{r}(x)}\right)}
$$

In both cases, as we enforce lossless condition, the total reflected power is equal to the incident one. The portion of the incident wave that does not couple in the desired direction because of the passive approximation and finite thickness of the system inevitably couples to other Floquet harmonics, indicated by the dashed lines in Fig. 3.

\section{APPENDIX D: COUPLING FROM PROPAGATING INTO GUIDED MODES: METASURFACE INFINITE COUPLERS}

Increasing the deflection angle beyond $\theta_{r}=90$ and towards complex angles is equivalent to coupling to a surface mode. Under this condition, the propagation constant of the scattered wave is purely imaginary along the normal $z$ axis and the lateral momentum of the guided mode is larger than the free-space wave number. For $\theta_{r}=90+j\left|\alpha_{r}\right|$ and under normal illumination, the incident and scattered fields can be generally expressed as 


$$
\begin{aligned}
\left(\mathbf{E}_{i}, \mathbf{H}_{i}\right) & =\left(\hat{y}, \frac{\hat{x}}{\eta_{0}}\right) E_{0} e^{j k_{0} z}, \\
\left(\mathbf{E}_{1 s}, \mathbf{H}_{1 s}\right) & =A_{r}\left(\hat{y}, \frac{\hat{x} \beta_{z}-\hat{z} \beta_{x}}{k_{0} \eta_{0}}\right) E_{0} e^{j \beta_{x} x} e^{j \beta_{z} z},
\end{aligned}
$$

with $\beta_{x}=-k_{0} \cosh \left(\alpha_{r}\right)$ and $\beta_{z}=j\left|\sqrt{\beta_{x}^{2}-k_{0}^{2}}\right|=j \sinh \left(\left|\alpha_{r}\right|\right)$. The net power absorbed by the surface is calculated as

$$
\begin{aligned}
\operatorname{Re}\left(-\bar{P}_{\text {surface }}\right) & =\operatorname{Re}\left[\frac{1}{2 X} \int_{X}\left(Y_{e}^{*}\left|\mathbf{E}_{t}\right|^{2}+Z_{m}\left|\mathbf{H}_{t}\right|^{2}\right) d x\right] \\
& =\frac{E_{0}^{2}}{2 \eta_{0}}
\end{aligned}
$$

with $X=2 \pi / \beta_{x}$. The graded surface absorbs the entire incident power independent of the amplitude of the scattered bound states $A_{r}$, consistent with the power conservation. To ensure passivity of the gradient metasurface, the reflection amplitude $\left|A_{r}\right|$ is chosen so that the local emerging power on the surface is always negative; i.e., $\forall x$, $\operatorname{Re}\left(\hat{z} \cdot \mathbf{P}_{\text {total }}\right)_{z \rightarrow 0^{+}}<0$. Following Eq. (D1), the local emerging power on the surface can be simplified as

$$
\begin{aligned}
& \operatorname{Re}\left(\hat{z} \cdot \mathbf{P}_{\text {total }}\right)_{z \rightarrow 0^{+}} \\
& \quad=-\frac{E_{0}^{2}}{4 \pi \eta_{0}}\left\{2 \pi+\left|A_{r}\right| \beta_{x} \lambda_{0} \sin \left[\angle A_{r}+\beta_{x} x-\tan ^{-1}\left(k_{0} /\left|\beta_{z}\right|\right)\right]\right\} .
\end{aligned}
$$

Enforcing $\operatorname{Re}\left(\hat{z} \cdot \mathbf{P}_{\text {total }}\right)_{z \rightarrow 0^{+}}<0$, the maximum tolerable reflection amplitude is found to be $\left|A_{r}\right|_{\max }=k_{0} / \beta_{x}$.

\section{APPENDIX E: PLANAR LENS}

As the final example, we discuss in the main text planar lenses designed based on our rigorous solution of wave equation to convert plane waves with flat phase fronts into cylindrical waves, in order to focus the incident energy. An optimal scattered wave can be considered as the time reverse of the wave radiated by a line source (point source in a two-dimensional lens), and fields take the general format [64]

$$
\begin{aligned}
\left(\mathbf{E}_{i}, \mathbf{H}_{i}\right)= & \left(\hat{y}, \frac{\hat{x}}{\eta_{0}}\right) E_{0} e^{j k_{0} z}, \\
\mathbf{E}_{1 s}= & \frac{-I_{0} k_{0} \eta_{0}}{4} H_{0}^{(1)}\left[k_{0} \sqrt{x^{2}+(z-f)^{2}}\right] \hat{y}, \\
\mathbf{H}_{1 s}= & \frac{-j I_{0} k_{0}}{4} H_{1}^{(1)}\left[k_{0} \sqrt{x^{2}+(z-f)^{2}}\right] \\
& \times\left(\frac{\hat{x}(z-f)}{\sqrt{x^{2}+(z-f)^{2}}}-\frac{\hat{z} x}{\sqrt{x^{2}+(z-f)^{2}}}\right) .
\end{aligned}
$$

We note that the field distributions in Eq. (E1) are accurate if an ideal drain, i.e., the time-reversed equivalent of the line source, is also positioned in the focal point. In practice, the absence of such a point may reduce the overall performance of the designed lens.

The metasurface lens is placed in the $x z$ plane with the focal point at $z=f$, and $H_{n}^{(1)}[r]$ refers to the Hankel function of type 1 with order $n$. Assuming a finite length of $2 L$ for the lens, power conservation determines the relation between $E_{0}$ and $I_{0}$, so as the surface on the whole is lossless,

$$
\begin{aligned}
-\left|\mathbf{P}_{\text {incident }}\right| & =-\frac{E_{0}^{2}}{2 \eta_{0}} 2 L, \\
\left|\mathbf{P}_{\text {reflected }}\right| & =\frac{\tan ^{-1}(L / f)}{\pi}\left(\text { power radiated by current } I_{0}\right) \\
& =\frac{I_{0}^{2} k_{0} \eta_{0}}{8 \pi} \tan ^{-1}(L / f),
\end{aligned}
$$

which simplifies to the relation $\left|E_{0}\right|=\left|I_{0}\right| \eta_{0} \times$ $\sqrt{\left[\tan ^{-1}(L / f) / \pi\right]\left(k_{0} / 8 L\right)}$. The relative phase of the fields is determined by the focusing properties of the surface. In fact, any relative phase between $E_{0}$ and $I_{0}, \alpha=\angle E_{0}-\angle I_{0}$, transforms the incident plane wave into the cylindrical wave with the corresponding phase. However, since we are working in reflection, we want to create a hot spot for the total field, and not only the scattered field. This means that, for a given $\alpha$, the scattered cylindrical beam can constructively or destructively add to the incident wave at the focal point. To achieve the sharpest focus, we theoretically predict the optimal phase difference for the design process. The total power flowing toward the focal point in its vicinity equals $P_{\kappa}=\left.\hat{\kappa} \cdot \mathbf{P}_{\text {total }}(x=\delta \cos \gamma, z=f+\delta \sin \gamma)\right|_{\delta \rightarrow 0,-\pi<\gamma<0}$, in which we define $\hat{\kappa}$ as the radial unit vector around the focal point. After some mathematical simplification and replacing Hankel functions by their approximation around zero, the derivative of $P_{\kappa}$ versus $\alpha$ is found to be proportional to $\left(d P_{\kappa} / d \alpha\right) \propto-4 \sin \left(k_{0} f+\alpha\right)$. The local power toward the focal point is then maximized for $\alpha=\pi-k_{0} f$. This optimum value of $\alpha$ is valid for a lossy or gainy metasurface lens, and when we approximate the surface as lossless (by ignoring the amplitude modulation), the best value of $\alpha$ changes. To account for this lossless approximation, the finite thickness of the lens, and also the absence of a sink at the focal point, in Fig. 9 we tune this relative phase in Eq. (E1) to maximize the total power at the focal point.

[1] J. D. Joannopoulos et al. Photonic Crystals: Molding the Flow of Light (Princeton University Press, Princeton, NJ, 2011).

[2] N. Engheta and R.W. Ziolkowski, Metamaterials: Physics and Engineering Explorations (John Wiley \& Sons, New York, 2006).

[3] J. B. Pendry, Negative Refraction Makes a Perfect Lens, Phys. Rev. Lett. 85, 3966 (2000). 
[4] P. Russell, Photonic Crystal Fibers, Science 299, 358 (2003).

[5] S. L. McCall, P. Platzman, R. Dalichaouch, D. Smith, and S. Schultz, Microwave Propagation in Two-Dimensional Dielectric Lattices, Phys. Rev. Lett. 67, 2017 (1991).

[6] D. Schurig, J. J. Mock, B. J. Justice, S. A. Cummer, J. B. Pendry, A. F. Starr, and D. R. Smith, Metamaterial Electromagnetic Cloak at Microwave Frequencies, Science 314, 977 (2006).

[7] U. Leonhardt, Optical Conformal Mapping, Science 312, 1777 (2006).

[8] J. B. Pendry, D. Schurig, and D. R. Smith, Controlling Electromagnetic Fields, Science 312, 1780 (2006).

[9] S. Maci, G. Minatti, M. Casaletti, and M. Bosiljevac, Metasurfing: Addressing Waves on Impenetrable Metasurfaces, IEEE Antennas Wireless Propag. Lett. 10, 1499 (2011).

[10] A. A. Houck, J. B. Brock, and I. L. Chuang, Experimental Observations of a Left-Handed Material That Obeys Snell's Law, Phys. Rev. Lett. 90, 137401 (2003).

[11] R. E. Munson, H. A. Haddad, and J. W. Hanlen, U.S. Patent No. 4684952 (1987).

[12] D. Pozar, Flat Lens Antenna Concept Using Aperture Coupled Microstrip Patches, Electron. Lett. 32, 2109 (1996).

[13] D. M. Pozar, S. D. Targonski, and H. Syrigos, Design of Millimeter Wave Microstrip Reflectarrays, IEEE Trans. Antennas Propag. 45, 287 (1997).

[14] E. F. Kuester, M. A. Mohamed, M. Piket-May, and C. L. Holloway, Averaged Transition Conditions for Electromagnetic Fields at a Metafilm, IEEE Trans. Antennas Propag. 51, 2641 (2003).

[15] C. L. Holloway, E. F. Kuester, J. A. Gordon, J. O'Hara, J. Booth, and D. R. Smith, An Overview of the Theory and Applications of Metasurfaces: The Two-Dimensional Equivalents of Metamaterials, IEEE Antennas Propag. Mag. 54, 10 (2012).

[16] A. Papakostas, A. Potts, D. M. Bagnall, S. L. Prosvirnin, H. J. Coles, and N. I. Zheludev, Optical Manifestations of Planar Chirality, Phys. Rev. Lett. 90, 107404 (2003).

[17] Y. Zhao and A. Alu, Manipulating Light Polarization with Ultrathin Plasmonic Metasurfaces, Phys. Rev. B 84, 205428 (2011).

[18] Y. Zhao, N. Engheta, and A. Alù, Homogenization of Plasmonic Metasurfaces Modeled as Transmission-Line Loads, Metamaterials 5, 90 (2011).

[19] L. Novotny, Effective Wavelength Scaling for Optical Antennas, Phys. Rev. Lett. 98, 266802 (2007).

[20] N. Yu, P. Genevet, M. A. Kats, F. Aieta, J.-P. Tetienne, F. Capasso, and Z. Gaburro, Light Propagation with Phase Discontinuities: Generalized Laws of Reflection and Refraction, Science 334, 333 (2011).

[21] X. Ni, N. K. Emani, A. V. Kildishev, A. Boltasseva, and V. M. Shalaev, Broadband Light Bending with Plasmonic Nanoantennas, Science 335, 427 (2012).

[22] F. Aieta, P. Genevet, N. Yu, M. A. Kats, Z. Gaburro, and F. Capasso, Out-of-Plane Reflection and Refraction of Light by Anisotropic Optical Antenna Metasurfaces with Phase Discontinuities, Nano Lett. 12, 1702 (2012).
[23] C. Pfeiffer and A. Grbic, Metamaterial Huygens' Surfaces: Tailoring Wave Fronts with Reflectionless Sheets, Phys. Rev. Lett. 110, 197401 (2013).

[24] N. Mohammadi Estakhri and A. Alù, Manipulating Optical Reflections Using Engineered Nanoscale Metasurfaces, Phys. Rev. B 89, 235419 (2014).

[25] N. Yu and F. Capasso, Flat Optics with Designer Metasurfaces, Nat. Mater. 13, 139 (2014), and references therein.

[26] F. Monticone, N. M. Estakhri, and A. Alù, Full Control of Nanoscale Optical Transmission with a Composite Metascreen, Phys. Rev. Lett. 110, 203903 (2013).

[27] A. Pors, M. G. Nielsen, R. L. Eriksen, and S. I. Bozhevolnyi, Broadband Focusing Flat Mirrors Based on Plasmonic Gradient Metasurfaces, Nano Lett. 13, 829 (2013).

[28] C. L. Holloway, M. A. Mohamed, E. F. Kuester, and A. Dienstfrey, Reflection and Transmission Properties of a Metafilm: With an Application to a Controllable Surface Composed of Resonant Particles, IEEE Transactions on Electromagnetic Compatibility 47, 853 (2005).

[29] A. Epstein and G. V. Eleftheriades, Passive Lossless Huygens Metasurfaces for Conversion of Arbitrary Source Field to Directive Radiation, IEEE Trans. Antennas Propag. 62, 5680 (2014).

[30] S. Sun et al., High-Efficiency Broadband Anomalous Reflection by Gradient Meta-Surfaces, Nano Lett. 12, 6223 (2012).

[31] N. Mohammadi Estakhri, C. Argyropoulos, and A. Alù, Graded Metascreens to Enable a New Degree of Nanoscale Light Management, Phil. Trans. R. Soc. A 373, 20140351 (2015).

[32] C. Pfeiffer and A. Grbic, Bianisotropic Metasurfaces for Optimal Polarization Control: Analysis and Synthesis, Phys. Rev. Applied 2, 044011 (2014).

[33] M. Selvanayagam and G. Eleftheriades, Polarization Control Using Tensor Huygens Surfaces, IEEE Trans. Antennas Propag. 62, 6155 (2014).

[34] By optimal, here and in the following, we mean the surface that allows realizing conversion to the desired wave front with unitary efficiency.

[35] V. S. Asadchy, M. Albooyeh, S. N. Tcvetkova, A. DíazRubio, Y. Ra'di, and S. A. Tretyakov, Perfect Control of Reflection and Refraction Using Spatially Dispersive Metasurfaces, Phys. Rev. B 94, 075142 (2016).

[36] A. Epstein and G. V. Eleftheriades, Arbitrary PowerConserving Field Transformations with Passive Lossless Omega-Type Bianisotropic Metasurfaces, IEEE Trans. Antennas Propag. 64, 3880 (2016).

[37] C. M. Bender and S. Boettcher, Real Spectra in NonHermitian Hamiltonians Having $\mathcal{P} \mathcal{T}$ Symmetry, Phys. Rev. Lett. 80, 5243 (1998).

[38] C. E. Rüter, K. G. Makris, R. El-Ganainy, D. N. Christodoulides, M. Segev, and D. Kip, Observation of Parity-Time Symmetry in Optics, Nat. Phys. 6, 192 (2010).

[39] L. Feng, Y.-L. Xu, W. S. Fegadolli, M.-H. Lu, J. E. B. Oliveira, V. R. Almeida, Y.-F. Chen, and A. Scherer, Experimental Demonstration of a Unidirectional Reflectionless Parity-Time Metamaterial at Optical Frequencies, Nat. Mater. 12, 108 (2013).

[40] M. Kim, A. M. H. Wong, and G. V. Eleftheriades, Optical Huygens' Metasurfaces with Independent Control of the 
Magnitude and Phase of the Local Reflection Coefficients, Phys. Rev. X 4, 041042 (2014).

[41] M. A. Al-Joumayly and N. Behdad, A Generalized Method for Synthesizing Low-Profile, Band-Pass Frequency Selective Surfaces with Non-Resonant Constituting Elements, IEEE Trans. Antennas Propag. 58, 4033 (2010).

[42] M. Selvanayagam and G. V. Eleftheriades, Discontinuous Electromagnetic Fields Using Orthogonal Electric and Magnetic Currents for Wavefront Manipulation, Opt. Express 21, 14409 (2013).

[43] L. Zou, W. Withayachumnankul, C. M. Shah, A. Mitchell, M. Bhaskaran, S. Sriram, and C. Fumeaux, Dielectric Resonator Nanoantennas at Visible Frequencies, Opt. Express 21, 1344 (2013).

[44] L. Liu, Q. Lin, Y. Sun, T. Liu, C. Bao, F. Li, and L. Zhu Broadband Metasurfaces with Simultaneous Control of Phase and Amplitude, Adv. Mater. 26, 5031 (2014).

[45] A. Epstein and G. V. Eleftheriades, Floquet-Bloch Analysis of Refracting Huygens Metasurfaces, Phys. Rev. B 90, 235127 (2014).

[46] http://www.eccosorb.com.

[47] Chip inductors from Murata Manufacturing Co., and multilayer high- $Q$ capacitors from Johanson Technology.

[48] A. Alù, A. Salandrino, and N. Engheta, Coupling of Optical Lumped Nanocircuit Elements and Effects of Substrates, Opt. Express 15, 13865 (2007).

[49] D. Lin, P. Fan, E. Hasman, and M. L. Brongersma, Dielectric Gradient Metasurface Optical Elements, Science 345, 298 (2014).

[50] J. Sautter, I. Staude, M. Decker, E. Rusak, D. N. Neshev, I. Brener, and Y.S. Kivshar, Active Tuning of All-Dielectric Metasurfaces, ACS Nano 9, 4308 (2015).

[51] J. S. Gomez-Diaz et al., M. Tymchenko, and A. Alù, Hyperbolic Plasmons and Topological Transitions Over Uniaxial Metasurfaces, Phys. Rev. Lett. 114, 233901 (2015).

[52] F. J. Garcia de Abajo, Graphene Plasmonics: Challenges and Opportunities, ACS Photonics 1, 135 (2014).
[53] J. Cai et al. Atomically Precise Bottom-Up Fabrication of Graphene Nanoribbons, Nature (London) 466, 470 (2010).

[54] D. Taillaert, P. Bienstman, and R. Baets, Compact Efficient Broadband Grating Coupler for Silicon-on-Insulator Waveguides, Opt. Lett. 29, 2749 (2004).

[55] S. Sun, Q. He, S. Xiao, Q. Xu, X. Li, and L. Zhou, GradientIndex Meta-Surfaces as a Bridge Linking Propagating Waves and Surface Waves, Nat. Mater. 11, 426 (2012).

[56] J. Wang, S. Qu, H. Ma, Z. Xu, A. Zhang, H. Zhou, H. Chen, and Y. Li, High-Efficiency Spoof Plasmon Polariton Coupler Mediated by Gradient Metasurfaces, Appl. Phys. Lett. 101, 201104 (2012).

[57] F. Ding et al., in Proceedings of the 2014 Conference on Lasers and Electro-Optics (CLEO) (OSA, San Jose, 2014).

[58] A. Pors, M. G Nielsen, T. Bernardin, J.-C. Weeber, and S. I. Bozhevolnyi, Efficient Unidirectional PolarizationControlled Excitation of Surface Plasmon Polaritons, Light Sci. Appl. 3, e197 (2014).

[59] A. Baron, E. Devaux, J.-C. Rodier, J.-P. Hugonin, E. Rousseau, C. Genet, T. W. Ebbesen, and P. Lalanne, Compact Antenna for Efficient and Unidirectional Launching and Decoupling of Surface Plasmons, Nano Lett. 11, 4207 (2011).

[60] D. Taillaert, F. Van Laere, M. Ayre, W. Bogaerts, D. Van Thourhout, P. Bienstman, and R. Baets, Grating Couplers for Coupling between Optical Fibers and Nanophotonic Waveguides, Jpn. J. Appl. Phys. 45, 6071 (2006).

[61] F. Aieta, P. Genevet, M. A. Kats, N. Yu, R. Blanchard, Z. Gaburro, and F. Capasso, Aberration-Free Ultrathin Flat Lenses and Axicons at Telecom Wavelengths Based on Plasmonic Metasurfaces, Nano Lett. 12, 4932 (2012).

[62] R. F. Harrington, Time-Harmonic Electromagnetic Fields (John Wiley \& Sons, New York, 2001).

[63] G. M. Whitman and F. Schwering, Reciprocity Identity for Periodic Surface Scattering, IEEE Trans. Antennas Propag. 27, 252 (1979).

[64] C. A. Balanis, Advanced Engineering Electromagnetics (John Wiley \& Sons, New York, 1989). 